Annals of Mathematics, 158 (2003), 165-206

\title{
Groups acting properly on "bolic" spaces and the Novikov conjecture
}

\author{
By Gennadi Kasparov and Georges Skandalis
}

\begin{abstract}
We introduce a class of metric spaces which we call "bolic". They include hyperbolic spaces, simply connected complete manifolds of nonpositive curvature, euclidean buildings, etc. We prove the Novikov conjecture on higher signatures for any discrete group which admits a proper isometric action on a "bolic", weakly geodesic metric space of bounded geometry.
\end{abstract}

\section{Introduction}

This work has grown out of an attempt to give a purely $K K$-theoretic proof of a result of A. Connes and H. Moscovici ([CM], $[\mathrm{CGM}])$ that hyperbolic groups satisfy the Novikov conjecture. However, the main result of the present paper appears to be much more general than this. In the process of this work we have found a class of metric spaces which contains hyperbolic spaces (in the sense of M. Gromov), simply connected complete Riemannian manifolds of nonpositive sectional curvature, euclidean buildings, and probably a number of other interesting geometric objects. We called these spaces "bolic spaces".

Our main result is the following:

TheOREM 1.1. Novikov's conjecture on "higher signatures" is true for any discrete group acting properly by isometries on a weakly bolic, weakly geodesic metric space of bounded coarse geometry.

- The notion of a "bolic" and "weakly bolic" space is defined in Section 2, as well as the notion of a "weakly geodesic" space;

- bounded coarse geometry (i.e. bounded geometry in the sense of P. Fan; see $[\mathrm{HR}])$ is discussed in Section 3.

All conditions of the theorem are satisfied, for example, for any discrete group acting properly and isometrically either on a simply connected complete Riemannian manifold of nonpositive, bounded sectional curvature, or on a euclidean building with uniformly bounded ramification numbers. All condi- 
tions of the theorem are also satisfied for word hyperbolic groups, as well as for finite products of groups of the above classes. Note also that the class of geodesic bolic metric spaces of bounded geometry is closed under taking finite products (which is not true, for example, for the class of hyperbolic metric spaces).

The Novikov conjecture for discrete groups which belong to the above described classes was already proved earlier by different methods. In the present paper we give a proof valid for all these cases simultaneously, without any special arrangement needed in each case separately. Moreover, the class of bolic spaces is not a union of the above classes but probably is much wider. Although we do not have at the moment any new examples of bolic spaces interesting from the point of view of the Novikov conjecture, we believe they may be found in the near future.

In [KS2] we announced a proof of the Novikov conjecture for discrete groups acting properly, by isometries on geodesic uniformly locally finite bolic metric spaces. The complete proof was given in a preprint, which remained unpublished since we hoped to improve the uniform local finiteness condition. This is done in the present paper where uniform local finiteness is replaced by a much weaker condition of bounded geometry.

Our proof follows the main lines of [K2] and [KS1]: we construct a 'proper' $\Gamma$-algebra $\mathcal{A}$, a 'dual Dirac' element $\eta \in K K^{\Gamma}(\mathbf{C}, \mathcal{A})$ and a 'Dirac' element in $K K^{\Gamma}(\mathcal{A}, \mathbf{C})$. In the same way as in [KS1], the construction of the dual Dirac element relies on the construction of an element $\gamma \in K K_{\Gamma}(\mathbf{C}, \mathbf{C})$ (the JulgValette element in the case of buildings; cf. [JV]).

Here is an explanation of the construction of these ingredients:

The algebra $\mathcal{A}$ is constructed in the following way ( $(7)$ : We may assume that our bolic metric space $X$ is locally finite (up to replacing it by a subspace consisting of the preimages in $X$ of the centers of balls of radius $\delta$ covering $X / \Gamma)$. The assumption of bounded geometry is used to construct a 'good' $\Gamma$-invariant measure $\mu$ on $X$. Corresponding to the Hilbert space $\mathcal{H}=L^{2}(X, \mu)$ is a $C^{*}$-algebra $\mathcal{A}(\mathcal{H})$ constructed in $[\mathrm{HKT}]$ and [HK]; denote by $H$ the subspace of $\Lambda^{*}\left(\ell^{2}(X)\right)$ spanned by $e_{x_{1}} \wedge \cdots \wedge e_{x_{p}}$, where the set $\left\{x_{1}, \ldots, x_{p}\right\}$ has diameter $\leq N$ (here $N$ is a large constant appearing in our construction and related to bolicity); then $\mathcal{A}$ is a suitable proper subalgebra of $\mathcal{K}(H) \widehat{\otimes} \mathcal{A}(\mathcal{H})$.

The inclusion of $\mathcal{A}$ in $\mathcal{K}(H) \widehat{\otimes} \mathcal{A}(\mathcal{H})$ together with the Dirac element of $\mathcal{A}(\mathcal{H})$ constructed in $[\mathrm{HK}]$, gives us the Dirac element for $\mathcal{A}$.

The element $\gamma(\S 6)$ is given by an operator $F_{x}$ acting on the Hilbert space $H$ mentioned above, where $x \in X$ is a point chosen as the origin. The operator $F_{x}$ acts on $e_{x_{1}} \wedge \cdots \wedge e_{x_{p}}$ as Clifford multiplication by a unit vector $\phi_{S, x} \in \ell^{2}(X)$ where $S=\left\{x_{1}, \ldots, x_{p}\right\}$ and $\phi_{S, x}$ has support in a set $Y_{S, x}$ of points closest to $x$ among the points in $S$ or points which can be added to $S$ keeping the diameter of $S$ not greater than $N$. The bolicity condition is used here. Namely: 
We prove that if $y \in Y_{S, x}$, denoting by $T$ the symmetric difference of $S$ and $\{y\}$, we have $\phi_{S, x}=\phi_{T, x}$, which gives that $F_{x}^{2}-1 \in \mathcal{K}(H)$ (this uses half of the bolicity, namely condition $\left(\mathrm{B} 2^{\prime}\right)$ ).

Averaging over the radius of a ball centered at $x$ used in the construction of $\phi_{S, x}$ allows us to prove that $\lim _{S \rightarrow \infty}\left\|\phi_{S, x}-\phi_{S, y}\right\|=0$, whence $F_{x}-F_{y} \in \mathcal{K}(H)$ for any $x, y \in X$, which shows that $F_{x}$ is $\Gamma$-invariant up to $\mathcal{K}(H)$ (this uses condition (B1)).

In the same way as $\phi_{S, x}$, we construct a measure $\theta_{S, x}$ supported by the points of $S$ which are the most remote from $x$. This is used as the center for the 'Bott element' in the construction of the dual Dirac element (Theorem 7.3.a).

There are also some additional difficulties we have to deal with:

a) Unlike the case of buildings (and the hyperbolic case), we do not know anything about contractibility of the Rips complex. We need to use an inductive limit argument, discussed in Sections 4 and 5.

b) The Dirac element appears more naturally as an asymptotic $\Gamma$-morphism. On the other hand, since we wish to obtain the injectivity of the BaumConnes map in the reduced $C^{*}$-algebra, we need to use $K K$-theory. This is taken care of in Section 8.

Our main result on the Novikov conjecture naturally corresponds to the injectivity part of the Baum-Connes conjecture for the class of groups that we consider (see Theorem 5.2). We do not discuss the surjectivity part of the Baum-Connes conjecture (except maybe in Proposition 5.11). We can mention however that our result has already been used by V. Lafforgue in order to establish the Baum-Connes conjecture for a certain class of groups ([L]). On the other hand, M. Gromov has recently given ideas for construction of examples of discrete groups which do not admit any uniform embedding into a Hilbert space ([G1], [G2]). For these groups the surjectivity part of the Baum-Connes conjecture with coefficients fails ([HLS]).

The paper is organized as follows: in Sections 2-4 we introduce the main definitions. Sections 5-8 contain the mains steps of the proof. More precisely:

- Bolicity is defined in Section 2, where we prove that hyperbolic spaces and Riemannian manifolds of nonpositive sectional curvature are bolic.

- The property of bounded geometry is discussed in Section 3.

- Section 4 contains some preliminaries on universal proper $\Gamma$-spaces and Rips complexes.

- Section 5 gives the statement of our main result and a general framework of the proof. 
- Section 6 contains the construction of the $\gamma$-element.

- Finally, in Sections 7 and 8 we explain the construction of the $C^{*}$-algebra of a Rips complex, give the construction of the dual Dirac and Dirac elements in $K K$-theory, and finish the proof of our main result.

The reader is referred to [K2] for the main definitions related to the equivariant $K K$-theory, graded algebras, graded tensor products and for some related jargon: for example, $\Gamma$-algebras are just $C^{*}$-algebras equipped with a continuous action of a locally compact group $\Gamma, C(X)$-algebras are defined in [K2], 1.5, etc. Unless otherwise specified, all tensor products of $C^{*}$-algebras are considered with the minimal $C^{*}$-norm. All groups acting on $C^{*}$-algebras are supposed to be locally compact and $\sigma$-compact, all discrete groups - countable.

\section{2. "Bolicity"}

Let $\delta$ be a nonnegative real number. Recall that a map (not necessarily continuous) $f: X \rightarrow X^{\prime}$ between metric spaces $(X, d)$ and $\left(X^{\prime}, d^{\prime}\right)$ is said to be a $\delta$-isometry if for every pair $(x, y)$ of elements of $X$ we have $\mid d^{\prime}(f(x), f(y))-$ $d(x, y) \mid \leq \delta$. Also, the metric space $(X, d)$ is said to be $\delta$-geodesic if for every pair $(x, y)$ of points of $X$, there exists a $\delta$-isometry $f:[0, d(x, y)] \rightarrow X$ such that $f(0)=x, f(d(x, y))=y$.

Definition 2.1. The space $(X, d)$ is said to be weakly $\delta$-geodesic if for every pair $(x, y)$ of points of $X$, and every $t \in[0, d(x, y)]$ there exists a point $a \in X$ such that $d(a, x) \leq t+\delta$ and $d(a, y) \leq d(x, y)-t+\delta$. The point $a \in X$ is said to be a $\delta$-middle point of $x, y$ if $|2 d(x, a)-d(x, y)| \leq 2 \delta$ and $|2 d(y, a)-d(x, y)| \leq 2 \delta$. We will say that the space $(X, d)$ admits $\delta$-middle points if there exists a map $m: X \times X \rightarrow X$ such that for any $x, y \in X$, the point $m(x, y)$ is a $\delta$-middle point of $x, y$. The map $m$ will be called a $\delta$-middle point map.

Note that in the above definition of a weakly $\delta$-geodesic space, one can obviously take $t \in[-\delta, 0] \cup[d(x, y), d(x, y)+\delta]$ and $a=x$ or $a=y$. This will be useful in Section 6 . Also note that a $\delta$-geodesic space is weakly $\delta$-geodesic. In a weakly $\delta$-geodesic space, every pair of points admits a $\delta$-middle point.

Definition 2.2. We will say that a metric space $(X, d)$ is $\delta$-bolic if:

(B1) For all $r>0$, there exists $R>0$ such that for every quadruple $x, y, z, t$ of points of $X$ satisfying $d(x, y)+d(z, t) \leq r$ and $d(x, z)+d(y, t) \geq R$, we have $d(x, t)+d(y, z) \leq d(x, z)+d(y, t)+2 \delta$.

(B2) There exists a map $m: X \times X \rightarrow X$ such that for all $x, y, z \in X$ we have $2 d(m(x, y), z) \leq\left(2 d(x, z)^{2}+2 d(y, z)^{2}-d(x, y)^{2}\right)^{1 / 2}+4 \delta$. 
We will say that a metric space $(X, d)$ is weakly $\delta$-bolic if it satisfies the condition (B1) and the following condition:

$\left(\mathrm{B}^{\prime}\right)$ There exists a $\delta$-middle point map $m: X \times X \rightarrow X$ such that if $x, y, z$ are points of $X$, then $d(m(x, y), z)<\max (d(x, z), d(y, z))+2 \delta$. Moreover, for every $p \in \mathbf{R}_{+}$, there exists $N(p) \in \mathbf{R}_{+}$such that for all $N \in \mathbf{R}_{+}$, $N \geq N(p)$, if $d(x, z) \leq N, d(y, z) \leq N$ and $d(x, y)>N$ then $d(m(x, y), z)$ $<N-p$.

Condition (B2') is a property of "strict convexity" of balls. Bolic spaces are obviously weakly bolic (a point $m(x, y)$ satisfying condition (B2) is automatically a $2 \delta$-middle point of $x, y$; apply condition (B2) to $z=x$ and $z=y$ ).

Proposition 2.3. Any $\delta$-hyperbolic space admitting $\delta$-middle points is $3 \delta / 2$-bolic.

Proof. Let $(X, d)$ be a $\delta$-hyperbolic metric space. Condition (B1) is obviously satisfied.

Assume moreover that we have a $\delta$-middle point map $m: X \times X \rightarrow X$. Let $z \in X$. The hyperbolicity condition gives:

$$
\begin{aligned}
& d(z, m(x, y))+d(x, y) \\
& \quad \leq \sup \{d(y, z)+d(x, m(x, y)), d(x, z)+d(y, m(x, y))\}+2 \delta \\
& \quad \leq \sup \{d(x, z), d(y, z)\}+\frac{d(x, y)+2 \delta}{2}+2 \delta .
\end{aligned}
$$

Therefore,

$$
2 d(z, m(x, y)) \leq 2 \sup \{d(x, z), d(y, z)\}-d(x, y)+6 \delta .
$$

Now, if $s, t, u$ are nonnegative real numbers such that $|t-u| \leq s$, we have

$$
(2 t-u)^{2}+u^{2}=2 t^{2}+2(t-u)^{2} \leq 2 t^{2}+2 s^{2} .
$$

Setting $s=\inf \{d(x, z), d(y, z)\}, t=\sup \{d(x, z), d(y, z)\}$ and $u=d(x, y)$, we find

$$
2 \sup \{d(x, z), d(y, z)\}-d(x, y) \leq\left(2 d(x, z)^{2}+2 d(y, z)^{2}-d(x, y)^{2}\right)^{1 / 2} .
$$

Proposition 2.4. Every nonpositively curved simply connected complete Riemannian manifold is $\delta$-bolic for any $\delta>0$.

In particular Euclidean spaces, as well as symmetric spaces $G / K$, where $G$ is a semisimple Lie group and $K$ its maximal compact subgroup, are bolic.

Proof. Let us first prove (B2). Recall the cosine theorem for nonpositively curved manifolds (cf. [H, 1.13.2]): For any geodesic triangle with edges of length 
$a, b$ and $c$ and the angle between the edges of the length $a$ and $b$ equal to $\alpha$, one has:

$$
a^{2}+b^{2}-2 a b \cos \alpha \leq c^{2} .
$$

Define $m(x, y)$ as the middle point of the unique geodesic segment joining $x$ and $y$. Apply the cosine theorem to the two geodesic triangles: $(x, z, m(x, y))$ and $(y, z, m(x, y))$. If we put $a=d(x, z), b=d(y, z), c=d(x, m(x, y))=$ $d(y, m(x, y)), e=d(z, m(x, y))$ then

$$
c^{2}+e^{2}-2 c e \cos \alpha \leq a^{2}, \quad c^{2}+e^{2}-2 c e \cos (\pi-\alpha) \leq b^{2}
$$

where the angle of the first triangle opposite to the edge $(x, z)$ is equal to $\alpha$. The sum of these two inequalities gives (B2) with $\delta=0$.

For the proof of (B1), let $x$ and $y \in X$. Suppose that $z(s), 0 \leq s \leq$ $d(z, t)$, is a geodesic segment (parametrized by distance) joining $t=z(0)$ with $z=z(d(z, t))$. Then it follows from the cosine theorem that

$$
|(\partial / \partial s)(d(y, z(s))-d(x, z(s)))| \leq \frac{2 c}{a(s)+b(s)},
$$

where $c=d(x, y), a(s)=d(x, z(s)), b(s)=d(y, z(s))$.

Indeed, the norm of the derivative on the left-hand side does not exceed $\|\operatorname{grad} f(u)\|$, where $f(u)=d(x, u)-d(y, u)$ is a function of $u=z(s)$. It is clear that $\|\operatorname{grad} f(u)\|$ is the norm of the difference between the two unit vectors tangent to the geodesic segments $[x, u]$ and $[y, u]$ at the point $u$, so that $\|\operatorname{grad} f(u)\|^{2}=2(1-\cos \alpha)$, where $\alpha$ is the angle between these two vectors. The cosine theorem applied to the geodesic triangle $(x, y, u=z(s))$ gives: $a(s)^{2}+b(s)^{2}-c^{2} \leq 2 a(s) b(s) \cos \alpha$, whence $2 a(s) b(s)(1-\cos \alpha) \leq$ $c^{2}-(a(s)-b(s))^{2}$. Therefore,

$$
\|\operatorname{grad} f(u)\|^{2} \leq \frac{c^{2}-(a(s)-b(s))^{2}}{a(s) b(s)} \leq \frac{4 c^{2}}{(a(s)+b(s))^{2}}
$$

since $c \leq a(s)+b(s)$. This implies the above inequality.

Integrating this inequality over $s$, one gets the estimate:

$$
(d(y, z)-d(x, z))-(d(y, t)-d(x, t)) \leq \frac{2}{R-r} d(x, y) d(z, t)
$$

with $R$ and $r$ as in the condition (B1), which gives (B1) with $\delta$ arbitrarily small.

Proposition 2.5. Euclidean buildings are $\delta$-bolic for any $\delta>0$.

Proof. The property (B2) (with $\delta=0$ ) is proved in [BT, Lemma 3.2.1]. To prove (B1) let us denote the left side of $(1)$ by $q(x, y ; z, t)$. Then, clearly, $q(x, y ; z, t)+q(y, u ; z, t)=q(x, u ; z, t)$. The same type of additivity holds also 
in the $(z, t)$-variables. Now when the points $(x, y)$ are in one chamber and points $(z, t)$ in another one, we can apply the inequality (1) because in this case all four points $x, y, z, t$ belong to one apartment. In general we reduce the assertion to this special case by using the above additivity property.

Proposition 2.6. A product of two bolic spaces when endowed with the distance such that $d\left((x, y),\left(x^{\prime}, y^{\prime}\right)\right)^{2}=d\left(x, x^{\prime}\right)^{2}+d\left(y, y^{\prime}\right)^{2}$ is bolic.

Proof. Let $\left(X_{1}, d\right)$ and $\left(X_{2}, d\right)$ be two $\delta$-bolic spaces. We show that $X_{1} \times X_{2}$ is $2 \delta$-bolic. Take $r>0$ and let $R$ be the corresponding constant in the condition (B1) for both $X_{i}$. Let $R^{\prime} \in \mathbf{R}_{+}$be big enough. For $x_{i}, y_{i}, z_{i}, t_{i} \in X_{i}$, put $x=\left(x_{1}, x_{2}\right), y=\left(y_{1}, y_{2}\right), z=\left(z_{1}, z_{2}\right)$ and $t=\left(t_{1}, t_{2}\right)$. Assume that $d(x, y)+d(z, t) \leq r$ and $d(x, z)+d(y, t) \geq R^{\prime}$. We distinguish two cases:

- We have $d\left(x_{1}, z_{1}\right)+d\left(y_{1}, t_{1}\right) \geq R$ and $d\left(x_{2}, z_{2}\right)+d\left(y_{2}, t_{2}\right) \geq R$.

In this case

$$
d\left(x_{i}, t_{i}\right)+d\left(y_{i}, z_{i}\right) \leq d\left(x_{i}, z_{i}\right)+d\left(y_{i}, t_{i}\right)+2 \delta .
$$

Put

$$
z_{i}^{\prime}=d\left(x_{i}, z_{i}\right), y_{i}^{\prime}=d\left(x_{i}, t_{i}\right)-d\left(y_{i}, t_{i}\right), t_{i}^{\prime}=d\left(x_{i}, t_{i}\right) .
$$

Note that

$$
d\left(y_{i}, z_{i}\right) \leq d\left(x_{i}, z_{i}\right)+d\left(y_{i}, t_{i}\right)-d\left(x_{i}, t_{i}\right)+2 \delta=z_{i}^{\prime}-y_{i}^{\prime}+2 \delta .
$$

Note also that $\left|y_{i}^{\prime}\right| \leq d\left(x_{i}, y_{i}\right)$ and $\left|z_{i}^{\prime}-t_{i}^{\prime}\right| \leq d\left(z_{i}, t_{i}\right)$. Put $x^{\prime}=(0,0), y^{\prime}=$ $\left(y_{1}^{\prime}, y_{2}^{\prime}\right), z^{\prime}=\left(z_{1}^{\prime}, z_{2}^{\prime}\right)$ and $t^{\prime}=\left(t_{1}^{\prime}, t_{2}^{\prime}\right)$. As $\mathbf{R}^{2}$ is $\delta^{\prime}$-bolic for every $\delta^{\prime}$, if $R^{\prime}$ is large enough, we find that

$$
\left\|z^{\prime}-y^{\prime}\right\|+\left\|t^{\prime}-x^{\prime}\right\| \leq\left\|z^{\prime}-x^{\prime}\right\|+\left\|t^{\prime}-y^{\prime}\right\|+(4-2 \sqrt{2}) \delta .
$$

Now $\left\|z^{\prime}-x^{\prime}\right\|=d(x, z),\left\|t^{\prime}-x^{\prime}\right\|=d(x, t),\left\|t^{\prime}-y^{\prime}\right\|=d(y, t)$ and $d(y, z) \leq$ $\left\|y^{\prime}-z^{\prime}\right\|+2 \sqrt{2} \delta$. We therefore get condition (B1) in this case.

- We have $d\left(x_{2}, z_{2}\right)+d\left(y_{2}, t_{2}\right) \geq R$ but $d\left(x_{1}, z_{1}\right)+d\left(y_{1}, t_{1}\right) \leq R$.

Choosing $R^{\prime}$ large enough, we may assume that if $s, u \in \mathbf{R}_{+}$are such that $s \leq R+r$ and $\left(s^{2}+u^{2}\right)^{1 / 2} \geq R^{\prime} / 2-r$, then $\left(s^{2}+u^{2}\right)^{1 / 2} \leq u+\delta$. Therefore, $d(y, z) \leq d\left(y_{2}, z_{2}\right)+\delta$ and $d(x, t) \leq d\left(x_{2}, t_{2}\right)+\delta$, whence condition (B1) follows also in this case.

Let us check condition (B2). Let $x_{1}, y_{1}, z_{1} \in X_{1}$ and $x_{2}, y_{2}, z_{2} \in X_{2}$. Put $A_{i}=\left(2 d\left(x_{i}, z_{i}\right)^{2}+2 d\left(y_{i}, z_{i}\right)^{2}-d\left(x_{i}, y_{i}\right)^{2}\right)^{1 / 2}(i=1,2)$. We have

$$
\begin{aligned}
4\left(d\left(m_{1}\left(x_{1}, y_{1}\right), z_{1}\right)^{2}+d\left(m_{2}\left(x_{2}, y_{2}\right), z_{2}\right)^{2}\right) & \leq\left(A_{1}+4 \delta\right)^{2}+\left(A_{2}+4 \delta\right)^{2} \\
\leq & \left(\left(A_{1}^{2}+A_{2}^{2}\right)^{1 / 2}+4 \sqrt{2} \delta\right)^{2}
\end{aligned}
$$

and condition (B2) follows. 
Remark 2.7. Let $X$ be a $\delta$-bolic space, and let $Y$ be a subspace of $X$ such that for every pair $(x, y)$ of points of $Y$ the distance of $m(x, y)$ to $Y$ is $\leq \delta$. Then $Y$ is $2 \delta$-bolic. The same is true for weakly bolic spaces.

Remark 2.8. Bolicity is very much a euclidean condition. On the other hand, weak bolicity, is not at all euclidean. Let $E$ be a finite-dimensional normed space.

(a) If the unit ball of the dual space $E^{\prime}$ is strictly convex then $E$ satisfies condition (B1).

(b) If there are no segments of length 1 in the unit sphere of $E$, then $E$ satisfies condition $\left(\mathrm{B} 2^{\prime}\right)$.

Indeed, an equivalent condition for the strict convexity of the unit ball of $E^{\prime}$ is that for any nonzero $x \in E$, there exists a unique $\ell_{x}$ in the unit sphere of $E^{\prime}$ such that $\ell_{x}(x)=\|x\|$; moreover, the map $x \mapsto\|x\|$ is differentiable at $x$, its differential is $\ell_{x}$ and the map $x \mapsto \ell_{x}$ is continuous and homogeneous (i.e. $\ell_{\lambda x}=\ell_{x}$ for $\left.\lambda>0\right)$.

Now, let $r>0$. There exists an $\varepsilon>0$ such that for all $u, v \in E$ of norm 1 , if $\|u-v\| \leq \varepsilon$, then $\left\|\ell_{u}-\ell_{v}\right\| \leq \delta / r$. Take $x, y, z, t \in E$ satisfying $\|x-y\| \leq r,\|z-t\| \leq r$ and $\|x-z\| \geq 2 r / \varepsilon+r$. Note that for nonzero $u, v \in E$, we have \|\|$u\left\|^{-1} u-\right\| v\left\|^{-1} v\right\| \leq 2\|u-v\|\|u\|^{-1}$.

For every $s \in[0,1]$, set $x_{s}=s x+(1-s) y$. Since $\left\|x_{s}-z\right\| \geq 2 r / \varepsilon$, the distance between $u_{s}=\left\|x_{s}-z\right\|^{-1}\left(x_{s}-z\right)$ and $v_{s}=\left\|x_{s}-t\right\|^{-1}\left(x_{s}-t\right)$ is $\leq \varepsilon$. Therefore the derivative of $s \mapsto\left\|x_{s}-z\right\|-\left\|x_{s}-t\right\|$, which is equal to $\left(\ell_{u_{s}}-\ell_{v_{s}}\right)(x-y)$, is $\leq \delta$. Therefore condition (B1) is satisfied.

Assume now that there are no segments of length 1 in the unit sphere of $E$. Let $k=\sup \{\|y+z\| / 2,\|y\| \leq 1,\|z\| \leq 1\|y-z\| \geq 1\}$. By compactness and since there are no segments of length 1 in the unit sphere of $E, k<1$. If $x, y, z \in E$ satisfy $\|x-z\| \leq N,\|y-z\| \leq N$, and $\|x-y\| \geq N$, then $\|z-(x+y) / 2\| \leq k N$. Setting $m(x, y)=(x+y) / 2$ we obtain condition $\left(\mathrm{B}^{\prime}\right)$ because for any $p>0$ there is an $N>0$ such that $k N<N-p$.

Remark 2.9. It was proved recently by M. Bucher and A. Karlsson ([BK]) that condition (B2) actually implies (B1).

\section{Bounded geometry}

Consider a metric space $(X, d)$ which is proper in the sense that any closed bounded subset in $X$ is compact. Let us fix some notation:

For $x \in X$ and $r \in \mathbf{R}_{+}$, let $B(x, r)=\{y \in X, d(x, y)<r\}$ be the open ball with center $x$ and radius $r$ and $\bar{B}(x, r)=\{y \in X, d(x, y) \leq r\}$ the closed ball with center $x$ and radius $r$. 
The following condition of bounded coarse geometry will be important for us. Recall from $[\mathrm{HR}]$ its definition:

Definition 3.1. A metric space $X$ has bounded coarse geometry if there exists $\delta>0$ such that for any $R>0$ there exists $K=K(R)>0$ such that in any closed ball of radius $R$, the maximal number of points with pairwise distances between them $\geq \delta$ does not exceed $K$.

We need to consider a situation in which a locally compact group $\Gamma$ acts properly by isometries on $X$. For simplicity we will assume in this section that $\Gamma$ is a discrete group.

Proposition 3.2. Let $X$ be a proper metric space of bounded coarse geometry and $\Gamma$ a discrete group which acts properly and isometrically on $X$. Then there exists on $X$ a $\Gamma$-invariant positive measure $\mu$ with the property that for any $R>0$ there exists $K>0$ such that for any $x \in X, \mu(\bar{B}(x, R)) \leq K$ and $\mu(B(x, 2 \delta)) \geq 1$.

Proof. Let $Y$ be a maximal subset of points of $X$ such that the distance between any point of $Y$ and a $\Gamma$-orbit passing through any other point of $Y$ is $\geq \delta$; by maximality of $Y$, for any $x \in X, d(x, \Gamma \cdot Y)<\delta$. For $y \in Y$, let $n(y, \delta)$ be the number of points of $\Gamma y \cap B(y, \delta)$. Define a measure on $X$ by assigning to any point on the orbit $\Gamma y$ the mass $n(y, \delta)^{-1}$. In this way we define a $\Gamma$ invariant measure $\mu$ on the set $\Gamma \cdot Y$. Outside of this set, put $\mu$ to be 0 . Note that for any $z \in \Gamma \cdot Y, \mu(B(z, \delta))=1$.

For any $x \in X$, there exists $z \in \Gamma \cdot Y$ such that $d(x, z)<\delta$; hence $\mu(B(x, 2 \delta)) \geq \mu(B(z, \delta))=1$.

For any $x \in X$ and $R>0$, let $Z$ be a maximal subset of $\Gamma \cdot Y \cap \bar{B}(x, R)$, with pairwise distances between any two points $\geq \delta$. By definition, $Z$ has at most $K(R)$ points. Obviously $\Gamma \cdot Y \cap \bar{B}(x, R) \subset \bigcup_{z \in Z} B(z, \delta)$; therefore

$\mu(\bar{B}(x, R))=\mu(\Gamma \cdot Y \cap \bar{B}(x, R)) \leq \mu\left(\bigcup_{z \in Z} B(z, \delta)\right) \leq \sum_{z \in Z} \mu(B(z, \delta)) \leq K(R)$

The following converse to the above proposition can be used in order to give examples of bounded coarse geometric spaces.

Proposition 3.3. Assume that $X$ is a metric space equipped with a positive measure $\mu$ (not necessarily $\Gamma$-invariant) which satisfies the following condition: there exists $\delta$ such that for all $R>0$, there exists $\tilde{K}=\tilde{K}(R)>0$ such that for any $x \in X, \mu(B(x, R)) \leq \tilde{K}$ and $\mu(B(x, \delta / 2)) \geq 1$. Then $X$ is a bounded coarse geometric space. 
Proof. Let $y_{1}, \ldots, y_{p} \in \bar{B}(x, R)$ be points with pairwise distances $\geq \delta$. Then the balls $B\left(y_{1}, \delta / 2\right), \ldots, B\left(y_{p}, \delta / 2\right)$ do not intersect and are all contained in $B(x, R+\delta / 2)$. Therefore, according to our assumption, $\tilde{K}(R+\delta / 2) \geq$ $\mu(B(x, R+\delta / 2)) \geq p$.

We will call a discrete metric space $(X, d)$ locally finite if any ball contains only a finite number of points.

Remark 3.4. All locally finite metric spaces equipped with an isometric proper action of a discrete group $\Gamma$, which have only a finite number of orbits of $\Gamma$-action, have bounded coarse geometry (BCG). All complete Riemannian manifolds with sectional curvature bounded from below are BCG-spaces. (This follows from Rauch's comparison theorem together with the criterion given in Proposition 3.3, the measure $\mu$ is the one defined by the Riemannian metric.) Euclidean buildings with uniformly bounded ramification numbers are BCG-spaces. A finite product of BCG-spaces is a BCG-space. Bounded coarse geometry is obviously hereditary with respect to passing to subspaces. Together with the hereditary property of bolicity (see Remark 2.7 of the previous section), this gives a large number of examples of locally finite bolic metric spaces of bounded coarse geometry. We record this for future use in the following:

Proposition 3.5. In any bolic, weakly geodesic metric space of bounded coarse geometry equipped with an isometric proper action of a discrete group $\Gamma$, there exists a $\Gamma$-invariant, locally finite, bolic, weakly geodesic metric subspace

of bounded coarse geometry. The assertion remains true if we replace bolicity by weak bolicity.

\section{Rips complexes}

Before we state (in the next section) our main result, we would like to introduce one more technical tool which will play a crucial role in the proof. Recall from $[\mathrm{BCH}]$ that there exists a "universal example" $\underline{E} \Gamma$ for proper actions of a locally compact group $\Gamma$. We will give now its construction in a form suitable for our purposes.

Let $X$ be a locally compact metrizable $\sigma$-compact space. We will denote by $\mathfrak{M}$ the set of finite positive measures on $X$ with total mass contained in $(1 / 2,1]$, endowed with the topology of duality with the algebra of continuous functions with compact support. Clearly, $\mathfrak{M}=K-\frac{1}{2} K$ where $K$ is the set of finite positive measures on $X$ with total mass $\leq 1$. As $K$ is compact and $\mathfrak{M}$ is open in $K, \mathfrak{M}$ is locally compact.

Let $\Gamma$ be a locally compact group acting properly on the space $X$. Then $\Gamma$ acts naturally on $\mathfrak{M}$. The following lemma describes the main properties of this action: 
Lemma 4.1. a) The action of $\Gamma$ on $\mathfrak{M}$ is proper.

b) For every locally compact space $Z$ endowed with a proper action of $\Gamma$, there is a continuous equivariant map, unique up to equivariant homotopy, $Z \rightarrow \mathfrak{M}$.

Proof. a) For every continuous function with compact support $\varphi$ on $X$, such that $0 \leq \varphi \leq 1$, let $U_{\varphi}$ denote the set of measures $\lambda \in \mathfrak{M}$ such that $\lambda(\varphi)>1 / 2$. Clearly the sets $U_{\varphi}$ form an open covering of $\mathfrak{M}$. Moreover, if $\varphi$ and $\psi$ have disjoint supports, $U_{\varphi}$ and $U_{\psi}$ are disjoint; hence, for every continuous function $\varphi$ with compact support $K \subset X$, the set

$$
\left\{g \in \Gamma, g U_{\varphi} \cap U_{\varphi} \neq \emptyset\right\} \subset\{g \in \Gamma, g(K) \cap K \neq \emptyset\}
$$

is relatively compact in $\Gamma$.

b) Since $\mathfrak{M}$ is a convex set, any two maps $Z \rightarrow \mathfrak{M}$ can be joined by a linear homotopy. This proves uniqueness (up to homotopy).

Let us prove existence. First, assume that $X=\Gamma$ with the action by left translations. Let $c$ be a positive continuous cut-off function on $Z$. This means, by definition, that the support of $c$ has compact intersection with the saturation of any compact subset of $Z$ and, for every $z \in Z, \int_{\Gamma} c\left(g^{-1} z\right) d g=1$. For any $z \in Z$, consider the function on $\Gamma: g \mapsto c\left(g^{-1} z\right)$. The product of this function with the Haar measure on $\Gamma$ is a probability measure on $\Gamma$. The map $Z \longrightarrow \mathfrak{M}$ associating to $z$ this measure is equivariant.

In general, choosing $x \in X$, we get an equivariant map $\Gamma \rightarrow X: g \mapsto g x$; the corresponding map on measures is an equivariant map from the space of measures on $\Gamma$ to the corresponding space of measures on $X$.

It follows from Lemma 4.1 that the space $\mathfrak{M}$ associated with any proper $\Gamma$-space $X$ is equivariantly homotopy equivalent to the universal $\Gamma$-space $\underline{E} \Gamma$. However, the space $\mathfrak{M}$ is too big. We prefer to deal with some subspaces of this space.

For this, assume moreover that $X$ is endowed with a $\Gamma$-invariant metric. For $k \in \mathbf{R}_{+}$, let $\mathfrak{M}_{k} \subset \mathfrak{M}$ denote the set of probability measures on $X$ whose support has diameter $\leq k$. Note that, if every bounded set of $X$ is relatively compact, then for every $k \in \mathbf{R}_{+}, \mathfrak{M}_{k}$ is a closed subset of $\mathfrak{M}$, hence locally compact.

Indeed, a positive measure $\mu$ has support of diameter $\leq k$ if and only if $\mu(f) \mu(g)=0$ for every pair of functions $f, g \in C_{c}(X)$ such that the distance between their supports is $>k$. Therefore, the set $\mathfrak{N}_{k} \subset \mathfrak{M}$ of measures of support of diameter $\leq k$ is a closed subset of $\mathfrak{M}$. For any continuous function with compact support $\varphi$ on $X$, let $U_{\varphi}$ denote the set of positive measures $\lambda \in \mathfrak{M}$ such that $\lambda(\varphi)>1 / 2$. Let $0 \leq \varphi \leq 1$. If every bounded set of $X$ is relatively compact, there exists a $\psi \in C_{c}(X)$ such that $0 \leq \psi \leq 1$ and $\psi(x)=1$, for every $x \in X$ with distance $\leq k$ to the support of $\varphi$. Then, 
for $\mu \in U_{\varphi} \cap \mathfrak{N}_{k}$ we have $\|\mu\|=\mu(\psi)$. Since the sets $U_{\varphi} \cap \mathfrak{N}_{k}$ form an open covering of $\mathfrak{N}_{k}$, the set $\mathfrak{M}_{k}$ of probability measures in $\mathfrak{N}_{k}$ is a closed subset of $\mathfrak{N}_{k}$.

For any locally compact space $Z$ endowed with a proper action of $\Gamma$, such that the quotient $Z / \Gamma$ is compact, there exists a $k \in \mathbf{R}_{+}$and a continuous equivariant map $Z \rightarrow \mathfrak{M}_{k}$. Moreover, if $f_{0}$ and $f_{1}$ are two such maps, they are homotopic in some $\mathfrak{M}_{N}$ for $N \geq k$.

Let then $\mathfrak{M}^{\prime}$ be the telescope of the spaces $\mathfrak{M}_{k}$. Let $Z$ be a locally compact, $\sigma$-compact space endowed with a proper action of $\Gamma$. Choose a proper function $\varphi: Z / \Gamma \rightarrow \mathbf{R}_{+}$. There exist:

- an increasing sequence $k_{n} \in \mathbf{R}_{+}$,

- an equivariant map $f_{n}: \varphi^{-1}([0, n]) \rightarrow \mathfrak{M}_{k_{n}}$,

- a sequence $N_{n}$ with $N_{n} \geq k_{n+1}$,

- an equivariant homotopy $F_{n}: \varphi^{-1}([0, n]) \times[0,1] \rightarrow \mathfrak{M}_{N_{n}}$ joining $f_{n}$ and the restriction of $f_{n+1}$.

Let $\psi: \mathbf{R}_{+} \rightarrow \mathbf{R}_{+}$be a continuous increasing function such that $\psi(n) \geq N_{n}$. Set then $f(x)=\left(F_{n}(x, \varphi(x)-n+1), \psi \circ \phi(x)\right)$ if $\varphi(x) \in[n-1, n]$. This is a continuous equivariant map $f: Z \rightarrow \mathfrak{M}^{\prime}$.

Moreover, one may use the same construction for homotopies. It follows that $\mathfrak{M}^{\prime}$ satisfies the conclusion of Lemma 4.1.b) for $\mathfrak{M}$. Therefore, the spaces $\mathfrak{M}$ and $\mathfrak{M}^{\prime}$ are $\Gamma$-equivariantly homotopy equivalent.

For us, it will be sufficient to think of $\mathfrak{M}$ as of an inductive limit (in the sense of homotopy theory) of spaces $\mathfrak{M}_{k}$.

Assume, furthermore, that our space $X$ has bounded coarse geometry. Let $\mu$ be a $\Gamma$-invariant measure on $X$ such that for any $x \in X, \mu(B(x, \delta)) \geq 1$ and for any $R>0$ there exists $K(R)>0$ such that for any subset $S \subset X$ of diameter $\leq R, \mu(S) \leq K(R)$ (see Proposition 3.2).

Definition 4.2. For any $N \in \mathbf{R}_{+}$, define a linear map $\tau: \mathfrak{M}_{N} \rightarrow L^{2}(X ; \mu)$ by the formula: $\tau(\nu)=\int_{X} \chi_{B(x, \delta)} d \nu(x)$, where $\chi_{Z}$ is the characteristic function of the set $Z$ in $X$.

Lemma 4.3. a) Let $R \in \mathbf{R}_{+}$and $g$ be a bounded $\mu$-measurable function on $X$ such that the diameter of its support is $\leq R$. Then $\|g\|_{1} K(R)^{-1 / 2} \leq$ $\|g\|_{2} \leq\|g\|_{\infty} K(R)^{1 / 2}$.

b) The image $\tau\left(\mathfrak{M}_{N}\right)$ in $L^{2}(X ; \mu)$ is contained between the spheres of radii $K(N+2 \delta)^{-1 / 2}$ and $K(N+2 \delta)^{1 / 2}$.

c) If $X$ is locally finite, the map $\tau: \mathfrak{M}_{N} \rightarrow L^{2}(X ; \mu)-\{0\}$ is continuous and proper in the topology induced by the weak topology of $L^{2}(X ; \mu)$. Therefore $\tau\left(\mathfrak{M}_{N}\right)$ is a locally compact proper $\Gamma$-space. 
Proof. a) Let $\chi$ be the characteristic function of the support of $g$. Replacing $\mu$ by $\chi \cdot \mu$ does not change the $p$-norms of $g$. Now the total mass of $\chi \cdot \mu$ is $\leq K(R)$, and a) follows.

b) Let $\nu \in \mathfrak{M}_{N}$. As the total mass of $\nu$ is 1 , we deduce that $\|\tau(\nu)\|_{\infty} \leq 1$. Since the $\mu$-measure of any open ball of radius $\delta$ is $\geq 1$, we have: $\|\tau(\nu)\|_{1} \geq 1$. Now b) follows from a) because the support of $\tau(\nu)$ has diameter $\leq N+2 \delta$.

c) The continuity of $\tau$ is obvious since $X$ is discrete. If $\mu_{n}$ is a sequence converging to the point at infinity of the one point compactification of $\mathfrak{M}_{N}$, its support goes to infinity in $X$, and so does the support of $\tau\left(\mu_{n}\right)$. As $\left\|\tau\left(\mu_{n}\right)\right\|$ is bounded by b), $\tau\left(\mu_{n}\right)$ converges weakly to 0 .

Remark. In the case of a non locally finite $X$, assertion c) remains true if we replace $\chi_{B(x, \delta)}$ by a continuous approximation.

When the space $X$ is locally finite, each $\mathfrak{M}_{k}$ is a locally finite simplicial complex, called a Rips complex. Therefore $\mathfrak{M}$ may be considered as an inductive limit (in the sense of homotopy theory) of Rips complexes $\mathfrak{M}_{k}$.

We remark here that such simplicial presentation of $\mathfrak{M}$ exists for any countable discrete group: we may take $X=\Gamma$ and define the distance by means of a proper length function $\ell$; for example: let $\left(g_{n}\right)_{n \in \mathbf{N}}$ be a set of generators for $\Gamma$ and let $\ell(g)$ be the minimum of $\sum_{i=1}^{p}\left|r_{i}\right|\left(n_{i}+1\right)$ over all decompositions $g=g_{n_{1}}^{r_{1}} \ldots g_{n_{p}}^{r_{p}}$.

Remarks. a) For $r \in[0,1)$, the space of finite positive measures on $X$ with total mass contained in $(r, 1]$ is locally compact, but the action of $\Gamma$ on this space is proper if and only if $r \geq 1 / 2$.

b) Assume that $X$ is endowed with a $\Gamma$-invariant measure $\mu$. Another realization of the classifying space for proper actions is the set of nonnegative $L^{2}$-functions of norm in the interval $\left(2^{-1 / 2}, 1\right]$.

\section{Novikov's conjecture: an outline of our approach}

Let $\Gamma$ be a countable discrete group. There are several conjectures associated with the Novikov conjecture for $\Gamma$ (see $[\mathrm{K} 2,6.4])$. All these conjectures deal with the classifying space for free proper actions of $\Gamma$, usually denoted by $E \Gamma$. The so-called Strong Novikov Conjecture is the statement that a natural homomorphism $\beta: R K_{*}^{\Gamma}(E \Gamma)=R K_{*}(B \Gamma) \rightarrow K_{*}\left(C^{*}(\Gamma)\right)$ is rationally injective. It is known that this statement implies the Novikov conjecture for $\Gamma$. 
However, we prefer to deal with the universal space for proper actions $\underline{E} \Gamma$ instead of $E \Gamma$. In view of the discussion of the previous section, we can consider $\underline{E} \Gamma$ as a locally compact space.

As explained in [BCH], the group $R K_{*}^{\Gamma}(E \Gamma) \otimes \mathbf{Q}$ is a subgroup of $R K_{*}^{\Gamma}(\underline{E} \Gamma)$ $\otimes$ Q. Also in $[\mathrm{BCH}]$, there is defined a natural homomorphism $R K_{*}^{\Gamma}(\underline{E} \Gamma) \rightarrow$ $K_{*}\left(C^{*}(\Gamma)\right.$ ), which we still prefer to call $\beta$ (we define this map below), and which rationally coincides on $R K_{*}^{\Gamma}(E \Gamma)$ with the above homomorphism $\beta$.

Let us fix some notation related with crossed products. Let $\Gamma$ be a locally compact group acting (on the left) on a $C^{*}$-algebra $B$. Denote by $d g$ the left Haar measure of $\Gamma$. The algebra $B$ is contained in the multiplier algebra of the crossed product $C^{*}(\Gamma, B)$ and there is a canonical strictly continuous morphism $g \mapsto u_{g}$ from $\Gamma$ to the unitary group of the multiplier algebra of the crossed product $C^{*}(\Gamma, B)$. For $b \in B$ and $g \in \Gamma$, we have $u_{g} b u_{g}^{*}=g \cdot b$; moreover, if $F \in C_{c}(\Gamma, B)$, the multiplier $\int F(g) u_{g} d g$ is actually an element of $C^{*}(\Gamma, B)$, and these elements form a dense subalgebra of $C^{*}(\Gamma, B)$.

Let $\Gamma$ act properly (on the left) on a locally compact space $Y$. If the action of $\Gamma$ is free, the algebras $C_{0}(Y / \Gamma)$ and $C^{*}\left(\Gamma, C_{0}(Y)\right)$ are Morita equivalent. In general, we have only a Hilbert $C^{*}\left(\Gamma, C_{0}(Y)\right)$-module $E_{Y}$ and an isomorphism between $C_{0}(Y / \Gamma)$ and $\mathcal{K}\left(E_{Y}\right)$ (which is enough for our purposes). To define $E_{Y}$, consider $C_{c}(Y)$ as a left $\Gamma$-module. For any $h, h_{1}, h_{2} \in C_{c}(Y)$ and $f \in$ $C_{c}\left(\Gamma, C_{0}(Y)\right)$, put

$$
\begin{aligned}
h \cdot f & =\int_{\Gamma} g(h) \cdot g\left(f\left(g^{-1}\right)\right) \cdot \nu(g)^{-1 / 2} d g \in C_{c}(Y), \\
\left\langle h_{1}, h_{2}\right\rangle(g) & =\nu(g)^{-1 / 2} \overline{h_{1}} g\left(h_{2}\right) \in C_{c}\left(\Gamma, C_{0}(Y)\right) \quad(g \in \Gamma),
\end{aligned}
$$

where $\nu(g)$ is the modular function of $\Gamma$. One can easily check that $C_{c}(Y)$ is a submodule of the pre-Hilbert module $C_{c}\left(\Gamma, C_{0}(Y)\right) \subset C^{*}\left(\Gamma, C_{0}(Y)\right)$ (considered as a module over itself). The embedding $i$ is given by the formula: $i(h)(g)=\nu(g)^{-1 / 2} \cdot c^{1 / 2} \cdot g(h)$, where $c$ is a positive continuous cut-off function on $Y$ (this means, by definition, that the support of $c$ has compact intersection with the saturation of any compact subset of $Y$ and, for every $y \in Y$, $\left.\int_{\Gamma} c\left(g^{-1} y\right) d g=1\right)$. It follows that the above inner product on $C_{c}(Y)$ is positive, so we can take completion which will be denoted by $E_{Y}$.

One checks immediately that $\mathcal{K}\left(E_{Y}\right)$ is isomorphic to $C_{0}(Y / \Gamma)$ (acting by pointwise multiplication on $\left.C_{c}(Y)\right)$.

If $Y / \Gamma$ is compact, then $\mathcal{K}\left(E_{Y}\right) \simeq C(Y / \Gamma)$ is unital, so $E_{Y}$ is a finitely generated projective $C^{*}\left(\Gamma, C_{0}(Y)\right)$-module. Therefore $E_{Y}$ defines an element of $K_{0}\left(C^{*}\left(\Gamma, C_{0}(Y)\right)\right.$ which will be denoted by $\lambda_{Y}$. Let $f: Y_{1} \rightarrow Y_{2}$ be a continuous proper $\Gamma$-map between two proper locally compact $\Gamma$-spaces with compact quotient. We obviously have $\lambda_{Y_{1}}=f^{*}\left(\lambda_{Y_{2}}\right)$ (where $f^{*}: K_{0}\left(C^{*}\left(\Gamma, C_{0}\left(Y_{2}\right)\right)\right) \rightarrow$ $K_{0}\left(C^{*}\left(\Gamma, C_{0}\left(Y_{1}\right)\right)\right)$ is the map induced by $\left.f\right)$. 


\section{The Baum-Connes map $\beta$}

Let $Y$ be a proper locally compact $\Gamma$-space with compact quotient. Define $\beta_{Y}: K_{\Gamma}^{i}\left(C_{0}(Y)\right) \rightarrow K_{i}\left(C^{*}(\Gamma)\right)$ by $\beta_{Y}(x)=\lambda_{Y} \otimes_{C^{*}\left(\Gamma, C_{0}(Y)\right)} j_{\Gamma}(x)$. If $f: Y_{1} \rightarrow Y_{2}$ is a continuous proper $\Gamma$-map between two proper locally compact $\Gamma$-spaces with compact quotient, we obviously have $\beta_{Y_{1}}=\beta_{Y_{2}} \circ f_{*}$.

Definition 5.1. $\quad$ Let $\Gamma$ be a locally compact group acting properly on a locally compact space $Z$. Put $R K_{i}^{\Gamma}(Z)=\lim _{\longrightarrow} K_{\Gamma}^{i}\left(C_{0}(Y)\right)$, where the inductive limit is taken on $Y$ running over $\Gamma$-invariant closed subsets of $Z$ such that $Y / \Gamma$ is compact. The Baum-Connes map $\beta: R K_{*}^{\Gamma}(\underline{E} \Gamma) \rightarrow K_{*}\left(C^{*}(\Gamma)\right)$ is the map defined at the inductive limit level by the maps $\beta_{Y}$. Denote also by $\beta_{\text {red }}: R K_{*}^{\Gamma}(\underline{E} \Gamma) \rightarrow K_{*}\left(C_{\text {red }}^{*}(\Gamma)\right)$ the composition of $\beta$ with the $K$-theory map associated with the homomorphism $C^{*}(\Gamma) \rightarrow C_{\text {red }}^{*}(\Gamma)$.

This map coincides with the map $\mu$ defined in [BCH].

Moreover, if $A$ is a $\Gamma$-algebra, we set $R K_{i}^{\Gamma}(Z ; A)=\lim _{\longrightarrow} K K_{\Gamma}^{i}\left(C_{0}(Y), A\right)$, where the inductive limit is taken on $Y$ running over $\Gamma$-invariant closed subsets of $Z$ such that $Y / \Gamma$ is compact. One defines in the same way the BaumConnes map $\beta^{A}: R K_{*}^{\Gamma}(\underline{E} \Gamma ; A) \rightarrow K_{*}\left(C^{*}(\Gamma, A)\right)$ and $\beta_{\text {red }}^{A}: R K_{*}^{\Gamma}(\underline{E} \Gamma ; A) \rightarrow$ $K_{*}\left(C_{\text {red }}^{*}(\Gamma, A)\right)$.

Theorem 1.1 is a consequence of the following theorem, which is the main result of this paper:

TheOREM 5.2. For any discrete group $\Gamma$ acting properly by isometries on a weakly bolic, weakly geodesic metric space of bounded coarse geometry and every $\Gamma$-algebra $A$, the Baum-Connes map $\beta_{\text {red }}^{A}$ is injective.

It follows that $\beta^{A}$ is also injective. We will prove Theorem 5.2 in Sections 7 and 8.

Let $A$ and $B$ be $\Gamma$-algebras. For $x \in R K_{i}^{\Gamma}(Z ; A)$ and $y \in K K^{\Gamma}(A, B)$, one may form the $K K$-product $x \otimes_{A} y \in R K_{i}^{\Gamma}(Z ; A)$. One obviously has:

Proposition 5.3. Let $A$ and $B$ be $\Gamma$-algebras and $a \in K K^{\Gamma}(A, B)$. For $x \in R K_{*}^{\Gamma}(\underline{E} \Gamma ; A), \beta^{A}(x) \otimes_{C^{*}(\Gamma, A)} j_{\Gamma}(a)=\beta^{B}\left(x \otimes_{A} a\right)$. If $\beta^{B}$ is an isomorphism and if there exists an element $b \in K K^{\Gamma}(B, A)$ such that $a \otimes_{B} b=1_{A} \in$ $K K^{\Gamma}(A, A)$ then $\beta^{A}$ is an isomorphism. The same holds if $\beta^{A}$ and $\beta^{B}$ are replaced by $\beta_{\text {red }}^{A}$ and $\beta_{\text {red }}^{B}$.

\section{Descent isomorphism}

Let $\Gamma$ be a locally compact group, $Y$ be a proper $\Gamma$-space, not necessarily $\Gamma$-compact. Denote by $\Lambda_{Y}$ the element

$$
\left(E_{Y}, 0\right) \in \mathcal{R} K K\left(Y / \Gamma ; C_{0}(Y / \Gamma), C^{*}\left(\Gamma, C_{0}(Y)\right)\right) .
$$


THEOREM 5.4. Let $\Gamma$ be a locally compact group, $Y$ a proper $\Gamma$-space and $B$ a $\Gamma-C_{0}(Y)$-algebra. Then, for $i=0,1$, the map $x \mapsto \Lambda_{Y} \otimes_{C^{*}\left(\Gamma, C_{0}(Y)\right)} j_{\Gamma}(x)$ is an isomorphism

$$
\mathcal{R} K K_{\Gamma}^{i}\left(Y ; C_{0}(Y), B\right) \simeq \mathcal{R} K K^{i}\left(Y / \Gamma ; C_{0}(Y / \Gamma), C^{*}(\Gamma, B)\right) .
$$

If $Y / \Gamma$ is compact,

$$
\mathcal{R} K K_{\Gamma}^{i}\left(Y ; C_{0}(Y), B\right) \simeq K_{i}\left(C^{*}(\Gamma, B)\right) .
$$

Before we give the proof of this theorem, we want to state a result which will be used in the proof. This is a generalization of the stabilization theorem for Hilbert modules ([K1]) involving proper group actions. Some generalizations of this kind are already known (cf. [P, 2.9], for example).

Proposition 5.5. Let $\Gamma$ be a locally compact group, $Y$ a proper $\Gamma$-space and $B$ a $\Gamma-C_{0}(Y)$-algebra. Assume that the Hilbert module $\mathcal{E}$ over $B$ is countably generated. Then

$$
\mathcal{E} \oplus\left(\oplus_{1}^{\infty} L^{2}(\Gamma, B)\right) \simeq \oplus_{1}^{\infty} L^{2}(\Gamma, B) .
$$

Proof. This isomorphism can be obtained in three steps. First, we embed $\mathcal{E}$ in $L^{2}(\Gamma, \mathcal{E})$ as a direct summand using a cut-off function $c$ on $Y$ as follows: $e \mapsto f(g)=g(c)^{1 / 2} e$. (The projection $L^{2}(\Gamma, \mathcal{E}) \rightarrow \mathcal{E}$ is given by $f \mapsto$ $\int_{\Gamma} f(g) g(c)^{1 / 2} d g$.) Next, we use the usual infinite sum trick: $\mathcal{E} \oplus \mathcal{E}^{\perp} \oplus \mathcal{E} \oplus \mathcal{E}^{\perp} \oplus \ldots$, to show that $\mathcal{E} \oplus\left(\oplus_{1}^{\infty} L^{2}(\Gamma, \mathcal{E})\right) \simeq \oplus_{1}^{\infty} L^{2}(\Gamma, \mathcal{E})$. Finally, we use the stabilization theorem without group action to get

$$
L^{2}(\Gamma, \mathcal{E}) \oplus\left(\oplus_{1}^{\infty} L^{2}(\Gamma, B)\right) \simeq \oplus_{1}^{\infty} L^{2}(\Gamma, B)
$$

Proof of Theorem 5.4. Let $(\mathcal{E}, T) \in \mathcal{R} K K_{\Gamma}\left(Y ; C_{0}(Y), B\right)$ and let $C^{*}(\Gamma, \mathcal{E})$ be the Hilbert module over $C^{*}(\Gamma, B)$ defined in $[\mathrm{K} 2,3.8]$ (in fact, $C^{*}(\Gamma, \mathcal{E})=$ $\left.\mathcal{E} \otimes_{B} C^{*}(\Gamma, B)\right)$. Define the Hilbert module $\widetilde{\mathcal{E}}$ over $C^{*}(\Gamma, B)$ by setting

$$
\widetilde{\mathcal{E}}=E_{Y} \otimes_{C^{*}\left(\Gamma, C_{0}(Y)\right)} C^{*}(\Gamma, \mathcal{E}) .
$$

The Hilbert module $\widetilde{\mathcal{E}}$ can also be constructed as follows. Let $\mathcal{E}_{c}=$ $C_{c}(Y) \cdot \mathcal{E}$. For any $e, e_{1}, e_{2} \in \mathcal{E}_{c}$ and $f \in C_{c}(\Gamma, B)$, put

$$
\begin{aligned}
e \cdot f & =\int_{\Gamma} g(e) \cdot g\left(f\left(g^{-1}\right)\right) \cdot \nu(g)^{-1 / 2} d g \in \mathcal{E}_{c}, \\
\left\langle e_{1}, e_{2}\right\rangle(g) & =\nu(g)^{-1 / 2}\left(e_{1}, g\left(e_{2}\right)\right)_{\mathcal{E}} \in C_{c}(\Gamma, B),
\end{aligned}
$$

where $\nu(g)$ is the modular function of $\Gamma$. There is a natural map of the algebraic tensor product $C_{c}(Y) \otimes C_{c}(\Gamma, \mathcal{E})$ to $\mathcal{E}_{c}$ given by

$$
f \otimes e \mapsto \int_{\Gamma} \nu(s)^{-1 / 2} s^{-1}(f) s^{-1}(e(s)) d s
$$


which preserves the inner products and the right actions of $C_{c}(\Gamma, B)$. This map extends to an isomorphism of $\widetilde{\mathcal{E}}$ with the completion of $\mathcal{E}_{c}$.

An easy argument shows that $\mathcal{L}(\widetilde{\mathcal{E}})$ is isomorphic to the $\Gamma$-invariant part of $\mathcal{L}(\mathcal{E})$ and that $\mathcal{K}(\widetilde{\mathcal{E}})$ is isomorphic to $\mathcal{K}(\mathcal{E})^{\Gamma}$ (see [K2, Def. 3.2]). This means that we can consider $\widetilde{T}=\int_{\Gamma} g(c T) d g$ as an operator on $\widetilde{\mathcal{E}}$ (where $c$ is a cut-off function). The map $(\mathcal{E}, T) \mapsto(\widetilde{\mathcal{E}}, \widetilde{T})$ gives a homomorphism of $\mathcal{R} K K_{\Gamma}^{i}\left(Y ; C_{0}(Y), B\right)$ to $\mathcal{R} K K^{i}\left(Y / \Gamma ; C_{0}(Y / \Gamma), C^{*}(\Gamma, B)\right)$ which coincides with the homomorphism $x \mapsto \Lambda_{Y} \otimes_{C^{*}\left(\Gamma, C_{0}(Y)\right)} j_{\Gamma}(x)$.

To prove that this is an isomorphism we apply Proposition 5.5 which allows us to assume that our initial Hilbert $B$-module $\mathcal{E}$ is isomorphic to $\oplus_{1}^{\infty} L^{2}(\Gamma, B)$. To finish the proof, it is enough to show that in this case, $\widetilde{\mathcal{E}} \simeq \oplus_{1}^{\infty} C^{*}(\Gamma, B)$ as a Hilbert module over $C^{*}(\Gamma, B)$. Of course, we will take only one copy of $L^{2}(\Gamma, B)$ and prove that if $\mathcal{E} \simeq L^{2}(\Gamma, B)$ then $\widetilde{\mathcal{E}} \simeq C^{*}(\Gamma, B)$ as a Hilbert module over $C^{*}(\Gamma, B)$. To get this, it will be convenient to consider $L^{2}(\Gamma, B)$ with the right $\Gamma$-action: $g(f)\left(g_{1}\right)=\nu(g)^{1 / 2} g\left(f\left(g_{1} g\right)\right)$, instead of the usual left one. (The two $\Gamma$-actions, clearly, correspond to each other under the automorphism $f(g) \mapsto \nu(g)^{-1 / 2} f\left(g^{-1}\right)$ of $L^{2}(\Gamma, B)$.) With this convention, the desired isomorphism $\widetilde{\mathcal{E}} \simeq C^{*}(\Gamma, B)$ is given by the formula: $\tilde{e}(g) \mapsto g(\tilde{e}(g))$.

\section{Proper algebras}

Definition 5.6. A $\Gamma$-algebra is said to be proper if it is a $\Gamma-C_{0}(Z)$-algebra for some proper $\Gamma$-space $Z$.

Since every proper $\Gamma$-space maps equivariantly to $\underline{E} \Gamma$, a $\Gamma$-algebra is proper if and only if it is a $\Gamma-C_{0}(\underline{E} \Gamma)$-algebra.

The following proposition is a particular case of some results of [Tu, §5]. As some of the statements and proofs there are a little too imprecise, we prefer to give a complete proof here.

Proposition 5.7. Let $\Gamma$ be a second countable locally compact group, $X$ a second countable locally compact $\Gamma$-space and $A$ a nuclear $\Gamma$-algebra. Assume that the $\Gamma$-algebra $A \otimes C_{0}(X)$ is proper. Then the functor $B \longrightarrow$ $R K K^{\Gamma}(X ; A, B)$ is 'half exact'. (All algebras are assumed to be separable.)

This means that for every $\Gamma$-equivariant short exact sequence of $\Gamma$-algebras

$$
0 \rightarrow J \stackrel{i}{\longrightarrow} B \stackrel{q}{\longrightarrow} B / J \rightarrow 0
$$

the sequence

$$
R K K^{\Gamma}(X ; A, J) \stackrel{i_{*}}{\longrightarrow} R K K^{\Gamma}(X ; A, B) \stackrel{q_{*}}{\longrightarrow} R K K^{\Gamma}(X ; A, B / J)
$$

is exact in its middle term, from which it follows that we have a six term exact sequence. 
Proof. We follow the proof of [S, Prop. 3.1]. Let us state the intermediate Lemmas $(3.2-3.3$ of $[\mathrm{S}])$ in our context.

Lemma 5.8. Let $(\mathcal{E}, F)$ be an element in $R K K^{\Gamma}(X ; A, B)$. Put $\overline{\mathcal{E}}=$ $\mathcal{E} \widehat{\otimes}_{B} B / J$.

a) If $q_{*}(\mathcal{E}, F)$ is degenerate, then $(\mathcal{E}, F)$ is in the image of $i_{*}$.

b) An operator homotopy $\left(\overline{\mathcal{E}}, G_{t}\right)$ in $R K K^{\Gamma}(X ; A, B / J)$ with $G_{0}=F \widehat{\otimes} 1$ can be lifted to an operator homotopy $\left(\mathcal{E}, F_{t}\right)$ in $R K K^{\Gamma}(X ; A, B)$ with $F_{0}=F$.

The proof of these facts is the same as in the nonequivariant setting:

Proof. We have an exact sequence $0 \rightarrow \mathcal{K}\left(\mathcal{E}_{J}\right) \rightarrow \mathcal{K}(\mathcal{E}) \rightarrow \mathcal{K}(\overline{\mathcal{E}}) \rightarrow 0$, where $\mathcal{E}_{J}=\{\xi \in \mathcal{E},\langle\xi, \xi\rangle \in J\}$.

a) If $q_{*}(\mathcal{E}, F)$ is degenerate, $\left(\mathcal{E}_{J}, F\right)$ is an element in $R K K^{\Gamma}(X ; A, J)$ which, as an element of $R K K^{\Gamma}(X ; A, B)$, is homotopic to $(\mathcal{E}, F)$.

b) Let $\mathcal{A}($ resp. $\mathcal{B})$ be the set of $T \in \mathcal{L}(\mathcal{E})$ (resp. $T \in \mathcal{L}(\overline{\mathcal{E}}))$ such that for all $a \in C_{0}(X) \otimes A$, the commutator $[a, T]$ is compact and the function $g \mapsto a(g T-T)(g \in \Gamma)$ is norm-continuous with compact values. Let also $\mathcal{I}$ (resp. $\mathcal{J}$ ) be the set of $T \in \mathcal{A}$ (resp. $T \in \mathcal{B})$ such that for all $a \in C_{0}(X) \otimes A$, Ta is compact.

We claim that the morphism $\mathcal{A} / \mathcal{I} \rightarrow \mathcal{B} / \mathcal{J}$ is onto. Indeed, let $S \in \mathcal{B}$. Since the morphism $\hat{q}: \mathcal{L}(\mathcal{E}) \rightarrow \mathcal{L}(\overline{\mathcal{E}})$ is surjective, we can find $T \in \mathcal{L}(\mathcal{E})$ with image $S$. Averaging $S$ and $T$ with respect to a continuous cut-off function on $\Gamma$, we may assume that $S$ and $T$ are $\Gamma$-continuous (this changes $S$ by some element of $\mathcal{J})$. Let $D$ be the (separable) subalgebra of $\mathcal{L}(\mathcal{E})$ generated by $\mathcal{K}(\mathcal{E}), C_{0}(X, A)$ and the translates of $T$ by $\Gamma$. Set $D_{1}=D \cap$ ker $\hat{q}$. Now thanks to Theorem 1.4 of $[\mathrm{K} 2]$, one may construct a $\Gamma$-continuous, equivariant up to $\mathcal{K}\left(\mathcal{E}_{J}\right)$, element $M \in \mathcal{L}(\mathcal{E})$ which commutes with $A$ and $T$ up to $\mathcal{K}\left(\mathcal{E}_{J}\right)$, such that $0 \leq M \leq 1, M D_{1} \subset \mathcal{K}\left(\mathcal{E}_{J}\right)$ and $(1-M) \mathcal{K}(\mathcal{E}) \subset \mathcal{K}\left(\mathcal{E}_{J}\right)^{1}$. From the last inclusion, it follows that $1-M \in \operatorname{ker} \hat{q}$, whence $S=\hat{q}(M T)$. Now, the elements $[T, a], a(g T-T)$ belong to $D \cap \hat{q}^{-1}(\mathcal{K}(\overline{\mathcal{E}}))$. Note that an element $x \in D \cap \hat{q}^{-1}(\mathcal{K}(\overline{\mathcal{E}}))$ can be written as a sum $x=y+z$ where $y \in \mathcal{K}(\mathcal{E})$ and $z \in D_{1}$. Therefore $M x \in \mathcal{K}(\mathcal{E})$. It follows easily that $M T \in \mathcal{A}$.

Let $U$ (resp. $V$ ) denote the set of self-adjoint elements of degree 1 and square 1 in $\mathcal{A} / \mathcal{I}(\operatorname{resp} . \mathcal{B} / \mathcal{J})$. The map $U \rightarrow V$ obviously satisfies the homotopy lifting property. The result follows.

\footnotetext{
${ }^{1}$ According to [K2], $M$ can be chosen as an element $M_{0}$ of $\mathcal{L}\left(\mathcal{E}_{J}\right)$. If $\mathcal{K}$ is an ideal in a $C^{*}$ algebra $\mathcal{D}$, the algebra $\mathcal{M}(\mathcal{D}, \mathcal{K})$ of multipliers $T$ of $\mathcal{D}$ such that $T \mathcal{D}+\mathcal{D} T \subset \mathcal{K}$ embeds both in $\mathcal{M}(\mathcal{K})$ and $\mathcal{M}(\mathcal{D})$; take $M \in \mathcal{L}(\mathcal{E})$ such that $(1-M) \in \mathcal{M}\left(\mathcal{K}(\mathcal{E}), \mathcal{K}\left(\mathcal{E}_{J}\right)\right)$ with image $1-M_{0}$ in $\mathcal{M}\left(\mathcal{K}\left(\mathcal{E}_{J}\right)\right)=\mathcal{L}\left(\mathcal{E}_{J}\right)$.
} 
By Lemma 5.8, if $q_{*}(\mathcal{E}, F)$ is operator homotopic to a degenerate element, its class is in the image of $i_{*}$. Now, if the class of $q_{*}(\mathcal{E}, F)$ is 0 , there exists a degenerate element $\left(\mathcal{E}^{\prime}, F^{\prime}\right)$ in $R K K^{\Gamma}(X ; A, B / J)$ such that $q_{*}(\mathcal{E}, F) \oplus\left(\mathcal{E}^{\prime}, F^{\prime}\right)$ is operator homotopic to a degenerate element. Furthermore, if a degenerate element $\left(\mathcal{E}^{\prime \prime}, F^{\prime \prime}\right)$ of $R K K^{\Gamma}(X ; A, B / J)$ contains $\left(\mathcal{E}^{\prime}, F^{\prime}\right)$ as a direct summand, then obviously $q_{*}(\mathcal{E}, F) \oplus\left(\mathcal{E}^{\prime \prime}, F^{\prime \prime}\right)$ is operator homotopic to a degenerate element.

Therefore, to end the proof of our proposition we just need to prove the following analogue of Lemma 3.5 in $[\mathrm{S}]$ :

Lemma 5.9. For every degenerate element $\left(\mathcal{E}^{\prime}, F^{\prime}\right)$ in $R K K^{\Gamma}(X ; A, B / J)$, there exists a degenerate element $(\widetilde{\mathcal{E}}, \widetilde{F})$ in $R K K^{\Gamma}(X ; A, B)$ such that $\left(\widetilde{\mathcal{E}} \widehat{\otimes}_{B} B / J, \widetilde{F} \widehat{\otimes} 1\right)$ contains $\left(\mathcal{E}^{\prime}, F^{\prime}\right)$ as a direct summand.

Proof. A representation of $A \otimes C_{0}(X)$ is just a pair of commuting representations. Now, since the left and right actions of $C_{0}(X)$ have to be the same, the only difference between elements of $K K_{\Gamma}\left(A, B \otimes C_{0}(X)\right)$ and $R K K_{\Gamma}(X ; A, B)$ is the compactness requirements. The degenerate elements are the same. The representation of $A$ together with the element $F^{\prime}$ define a representation $A \widehat{\otimes} \mathcal{C}_{1} \rightarrow \mathcal{L}\left(\mathcal{E}^{\prime}\right)$. In other words, degenerate elements in $R K K_{\Gamma}(X ; A, B)$ are just equivariant $\left(A \widehat{\otimes} \mathcal{C}_{1}, C_{0}(X) \otimes B\right)$-bimodules.

Using an equivariant representation of $A \widehat{\otimes} \mathcal{C}_{1}$ on a separable Hilbert space $\mathcal{H}$, we may find an equivariant $\left(A \widehat{\otimes} \mathcal{C}_{1}, C_{0}(X) \otimes B / J\right)$-bimodule $\mathcal{E}^{\prime \prime}$ isomorphic to $\mathcal{H} \widehat{\otimes} C_{0}(X) \otimes B / J$. Then $\mathcal{E}^{\prime}$ is a direct summand in $\mathcal{E}^{\prime} \oplus \mathcal{E}^{\prime \prime}$. By the (nonequivariant) stabilization theorem of [K1], the $C_{0}(X) \otimes B / J$-module $\mathcal{E}^{\prime} \oplus \mathcal{E}^{\prime \prime}$ is isomorphic to $\mathcal{E}^{\prime \prime}$, whence $\mathcal{L}\left(\mathcal{E}^{\prime} \oplus \mathcal{E}^{\prime \prime}\right)$ is a quotient of $\mathcal{L}\left(\mathcal{H} \widehat{\otimes} C_{0}(X) \otimes B\right)$. Denote by $\pi: A \widehat{\otimes} \mathcal{C}_{1} \rightarrow \mathcal{L}\left(\mathcal{E}^{\prime} \oplus \mathcal{E}^{\prime \prime}\right)$ the left action. Since $A \widehat{\otimes} \mathcal{C}_{1}$ is nuclear, the map $\pi$ admits a completely positive lifing. Using the Stinespring construction of [K1], we find a Hilbert $C_{0}(X) \otimes B$-module $\overline{\mathcal{E}}$ and a representation $\pi^{\prime}: A \widehat{\otimes} \mathcal{C}_{1} \rightarrow \mathcal{L}(\overline{\mathcal{E}})$ such that $\pi^{\prime} \otimes 1=\pi$. Note moreover that the $C_{0}(X)$ $\otimes B$-module $\overline{\mathcal{E}}$ contains $\mathcal{H} \widehat{\otimes} C_{0}(X) \otimes B$ as a direct summand, and is therefore isomorphic to $\mathcal{H} \widehat{\otimes} C_{0}(X) \otimes B$. Consequently, there exists an action of $\Gamma$ on $\overline{\mathcal{E}}$.

Note that the action of $A \widehat{\otimes} \mathcal{C}_{1}$ on $\overline{\mathcal{E}}$ and the isomorphism $U$ of $\overline{\mathcal{E}} \widehat{\otimes}_{B} B / J$ with $\mathcal{E}^{\prime} \oplus \mathcal{E}^{\prime \prime}$ are not assumed to be $\Gamma$-equivariant. This is taken care of by tensoring with $L^{2}(\Gamma)$. Set $\widetilde{\mathcal{E}}=L^{2}(\Gamma) \otimes \overline{\mathcal{E}}$ as a $C_{0}(X) \otimes B-\Gamma$-module. The action $\tilde{\pi}$ of $A \widehat{\otimes} \mathcal{C}_{1}$ on $\widetilde{\mathcal{E}}$ is given by $(\tilde{\pi}(a) \xi)(g)=g \cdot\left(\pi^{\prime}\left(g^{-1} \cdot a\right)\left(g^{-1} \cdot \xi(g)\right)\right.$ $\left(a \in A \widehat{\otimes} \mathcal{C}_{1}, \xi \in \widetilde{\mathcal{E}}=L^{2}(\Gamma, \overline{\mathcal{E}}), g \in \Gamma\right)$. It is equivariant.

We claim that the $\left(A \widehat{\otimes} \mathcal{C}_{1}, C_{0}(X) \otimes B / J\right)$-bimodules $\widetilde{\mathcal{E}}$ and $\left(\mathcal{E}^{\prime} \oplus \mathcal{E}^{\prime \prime}\right) \otimes L^{2}(\Gamma)$ are isomorphic. The element $\widetilde{U} \in \mathcal{L}\left(\widetilde{\mathcal{E}} \widehat{\otimes}_{B} B / J, \mathcal{E}^{\prime} \otimes L^{2}(\Gamma)\right)$ given by $(\widetilde{U} \xi)(g)=$ $g \cdot\left(U\left(g^{-1} \cdot \xi(g)\right)\right.$ is $\Gamma$-invariant. Moreover, since the action of $A \widehat{\otimes} \mathcal{C}_{1}$ on $\mathcal{E}^{\prime} \oplus \mathcal{E}^{\prime \prime}$ is $\Gamma$-equivariant, $\widetilde{U}$ intertwines the actions of $A \widehat{\otimes} \mathcal{C}_{1}$.

We finally prove that the $\left(A \widehat{\otimes} \mathcal{C}_{1}, C_{0}(X) \otimes B / J\right)$-bimodule $\mathcal{E}^{\prime}$ is a direct summand of $\mathcal{E}^{\prime} \otimes L^{2}(\Gamma)$. 
Let $Y$ be a proper $\Gamma$-space such that $C_{0}(Y)$ acts in a nondegenerate way by central multipliers on $C_{0}(X) \otimes A$. Let $c: Y \rightarrow \mathbf{C}$ be a positive cutoff function. Let $\Gamma$ act by left translations on $\Gamma$ and diagonally on $C_{0}(Y) \otimes$ $L^{2}(\Gamma)$. Associated to $c$ is an isometry $V_{0}: C_{0}(Y) \rightarrow C_{0}(Y) \otimes L^{2}(\Gamma)$ given by $V_{0}(\xi)(y, g)=\xi(y) c\left(g^{-1} y\right)^{1 / 2}$, where $\xi \in C_{0}(Y)$ and $V_{0}(\xi) \in C_{0}(Y) \otimes L^{2}(\Gamma)$ is seen as a function of two variables $y \in Y$ and $g \in \Gamma$. One checks immediately that $V_{0}$ is a $\Gamma$-invariant element of $\mathcal{L}\left(C_{0}(Y), C_{0}(Y) \otimes L^{2}(\Gamma)\right)$ and $V_{0}^{*} V_{0}=1$. Now, write

$$
C_{0}(X) \otimes A \widehat{\otimes} \mathcal{C}_{1}=C_{0}(Y) \otimes_{C_{0}(Y)}\left(C_{0}(X) \otimes A \widehat{\otimes} \mathcal{C}_{1}\right)
$$

and

$$
C_{0}(X) \otimes A \widehat{\otimes} \mathcal{C}_{1} \otimes L^{2}(\Gamma)=\left(C_{0}(Y) \otimes L^{2}(\Gamma)\right) \otimes_{C_{0}(Y)}\left(C_{0}(X) \otimes A \widehat{\otimes} \mathcal{C}_{1}\right) ;
$$

let

$$
V \in \mathcal{L}\left(C_{0}(X) \otimes A \widehat{\otimes} \mathcal{C}_{1}, C_{0}(X) \otimes A \widehat{\otimes} \mathcal{C}_{1} \otimes L^{2}(\Gamma)\right)
$$

be $V_{0} \otimes 1$. Since the action of $C_{0}(Y)$ is central, $V$ intertwines the natural left actions of $A \widehat{\otimes} \mathcal{C}_{1}$.

It follows that the equivariant $\left(A \widehat{\otimes} \mathcal{C}_{1}, C_{0}(X) \otimes B\right)$-bimodule $\mathcal{E}^{\prime}$ is a direct summand of $\left(A \widehat{\otimes} \mathcal{C}_{1} \otimes L^{2}(\Gamma)\right) \widehat{\otimes}_{A \widehat{\otimes}} \mathcal{C}_{1} \mathcal{E}^{\prime} \simeq \mathcal{E}^{\prime} \otimes L^{2}(\Gamma)$ and therefore a direct summand of $\left(\mathcal{E}^{\prime} \oplus \mathcal{E}^{\prime \prime}\right) \otimes L^{2}(\Gamma) \simeq \widetilde{\mathcal{E}} \otimes_{C_{0}(X) \otimes B}\left(C_{0}(X) \otimes B / J\right)$. This ends the proof.

Remark 5.10. Let $\Gamma$ be a locally compact group, $X$ a locally compact $\Gamma$-space and $A, A^{\prime}$ nuclear $\Gamma$-algebras. Assume that the $\Gamma$-algebras $A \otimes C_{0}(X)$ and $A^{\prime} \otimes C_{0}(X)$ are proper. Let $0 \rightarrow J \rightarrow B \stackrel{q}{\longrightarrow} B / J \rightarrow 0$ be a short exact sequence of $\Gamma$-algebras and $u$ be an element in $R K K_{\Gamma}\left(X ; A, A^{\prime}\right)$. Denote by $\partial: R K K_{\Gamma}(X ; A, B / J) \rightarrow R K K_{\Gamma}^{1}(X ; A, J)$ and $\partial^{\prime}: R K K_{\Gamma}\left(X ; A^{\prime}, B / J\right) \rightarrow$ $R K K_{\Gamma}^{1}\left(X ; A^{\prime}, J\right)$ the connecting maps associated with the exact sequences. These connecting maps are obtained by composing the map $B(0,1) \rightarrow C_{q}$ and the inverse of the map $e: J \rightarrow C_{q}$ where $C_{q}=B[0,1) / J(0,1)$ is the cone of $q$. Therefore, for any $x \in R K K_{\Gamma}\left(X ; A^{\prime}, B / J\right)$ we have $\partial\left(u \otimes_{A^{\prime}} x\right)=u \otimes_{A^{\prime}} \partial^{\prime}(x)$.

Using now Corollary A.4 of the appendix, for any $\Gamma$-invariant closed subset $Y$ of $\underline{E} \Gamma$ and any $\Gamma$-algebra $B$, we obtain an isomorphism: $K K_{\Gamma}^{i}\left(C_{0}(Y), B\right)=$ $E_{\Gamma}^{i}\left(C_{0}(Y), B\right)$, and therefore $R K_{i}^{\Gamma}(\underline{E} \Gamma ; B)$ is equal to the group $E_{\Gamma}^{i}(\underline{E} \Gamma, B)$ (of [GHT]). Moreover, for any proper algebra $B, C^{*}(\Gamma, B)=C_{\text {red }}^{*}(\Gamma, B)$. This allows us to apply certain methods and results of [GHT] to $K K$-theory. In particular we obtain

Proposition 5.11 (cf. [GHT, Th. 13.1]). Assume that the $\Gamma$-algebra $B$ is proper. Then the Baum-Connes homomorphisms $\beta^{B}$ and $\beta_{\mathrm{red}}^{B}$ are split surjective. If the group $\Gamma$ is discrete, these homomorphisms are isomorphisms. 
Sketch of proof. Let us describe the inverse map:

Note that $K_{i}\left(C^{*}(\Gamma, B)\right)$ is the inductive limit of $K_{i}\left(C^{*}\left(\Gamma, C_{0}(U) B\right)\right)$ where $U$ runs over open $\Gamma$-invariant subsets of $\underline{E} \Gamma$ such that $U / \Gamma$ is relatively compact.

Let $U \subset Y \subset \underline{E} \Gamma$ be $\Gamma$-invariant subsets of $\underline{E} \Gamma$ with $U$ open and $Y / \Gamma$ compact. Theorem 5.4 gives an isomorphism

$$
K_{i}\left(C^{*}\left(\Gamma, C_{0}(U) B\right)\right) \simeq \mathcal{R} K K_{\Gamma}^{i}\left(Y ; C_{0}(Y), C_{0}(U) B\right) .
$$

Denote by $\alpha_{U, Y}$ the composition

$$
K_{i}\left(C^{*}\left(\Gamma, C_{0}(U) B\right)\right) \simeq \mathcal{R} K K_{\Gamma}^{i}\left(Y ; C_{0}(Y), C_{0}(U) B\right) \rightarrow K K_{\Gamma}^{i}\left(C_{0}(Y), C_{0}(U) B\right)
$$

Let $U \subset V \subset Y \subset Z$ be $\Gamma$-invariant subsets of $\underline{E} \Gamma$ with $U, V$ open and $Y / \Gamma, Z / \Gamma$ compact. Denote by $u: C_{0}(U) B \rightarrow C_{0}(V) B$ the natural inclusion map and $q: C_{0}(Z) \rightarrow C_{0}(Y)$ the restriction map. We obviously have $q^{*} \circ \alpha_{U, Y}=\alpha_{U, Z}$ and $u_{*} \circ \alpha_{U, Y}=\alpha_{V, Y} \circ u_{*}$, from which it follows that we may take an inductive limit in $Y$ and get a map $\alpha_{U}: K_{i}\left(C^{*}\left(\Gamma, C_{0}(U) B\right)\right) \rightarrow$ $R K_{i}^{\Gamma}(\underline{E} \Gamma, B)$. Moreover, since $\alpha_{U}=\alpha_{V} \circ u_{*}$, the maps $\alpha_{U}$ define a morphism $\alpha: K_{i}\left(C^{*}(\Gamma, B)\right) \rightarrow R K_{i}^{\Gamma}(\underline{E} \Gamma, B)$.

It is easy to see that $\beta^{B} \circ \alpha$ is the identity of $K_{i}\left(C^{*}(\Gamma, B)\right)$. But the fact that the composition $\alpha \circ \beta^{B}$ is also the identity in the case of a discrete group $\Gamma$ is more complicated (cf. [GHT]).

\section{Sufficient conditions for the injectivity of the Baum-Connes map}

To establish the injectivity of the Baum-Connes map, we will use the following simple result, in which $\widehat{\otimes}$ stands for (graded) minimal or maximal tensor products:

Lemma 5.12. Let $\Gamma$ be a locally compact group and $B$ a $\Gamma$-algebra. Assume that for every closed $\Gamma$-invariant subset $Y \subset \underline{E} \Gamma$ with compact quotient there exist a $\Gamma$-algebra $A$ and elements $\eta \in K K_{i}^{\Gamma}(\mathbf{C}, A)$ and $d \in K K_{i}^{\Gamma}(A, \mathbf{C})$, such that $\beta^{A \widehat{\otimes} B}\left(\right.$ resp. $\left.\beta_{\text {red }}^{A \widehat{\otimes} B}\right)$ is injective and $p_{Y}^{*}\left(\eta \otimes_{A} d\right)=1_{Y}$, where $p_{Y}$ is the map $Y \rightarrow$ point and $p_{Y}^{*}$ is the map $K K^{\Gamma}(\mathbf{C}, \mathbf{C}) \rightarrow R K_{\Gamma}^{0}(Y)$. Then the Baum-Connes map $\beta^{B}$ (resp. $\beta_{\text {red }}^{B}$ ) is injective.

Proof. Indeed, let $z \in \operatorname{ker} \beta^{B}$; there exists $Y$ and there is a

$$
y \in K K_{*}^{\Gamma}\left(C_{0}(Y), B\right)
$$

with image $z$. Take $A, \eta, d$ corresponding to $Y$. As $z$ is in the image of $K K_{*}^{\Gamma}\left(C_{0}(Y), B\right)$, we have $z=z \otimes_{\mathbf{C}} \eta \otimes_{A} d$. Then $\beta^{A \widehat{\otimes} B}\left(z \otimes_{\mathbf{C}} \eta\right)=\beta^{B}(z) \otimes_{C^{*}(\Gamma, B)}$ $j_{\Gamma}\left(\sigma_{B}(\eta)\right)=0$, hence $z \otimes_{\mathbf{C}} \eta=0$ and $z=z \otimes_{\mathbf{C}} \eta \otimes_{A} d=0$.

Combining Lemma 5.12 and Proposition 5.11, we get:

Proposition 5.13. Assume that the group $\Gamma$ is discrete, and for every closed $\Gamma$-invariant subset $Y \subset \underline{E} \Gamma$ with compact quotient there exist 
- a proper $\Gamma$-algebra $\mathcal{A}$;

- elements $\eta \in K K_{i}^{\Gamma}(\mathbf{C}, \mathcal{A})$ and $d \in K K_{i}^{\Gamma}(\mathcal{A}, \mathbf{C})$ such that $p_{Y}^{*}\left(\eta \otimes_{\mathcal{A}} d\right)$ $=1_{Y}$, where $p_{Y}$ is the map $Y \rightarrow$ point, $p_{Y}^{*}$ is the $\operatorname{map} K K^{\Gamma}(\mathbf{C}, \mathbf{C}) \rightarrow$ $R K_{\Gamma}^{0}(Y)$.

Then $\beta_{\mathrm{red}}^{B}$ is injective for every $\Gamma$-algebra $B$.

Proof. Let $B$ be a $\Gamma$-algebra. The algebra $\mathcal{A} \widehat{\otimes} B$ is proper. By Proposition $5.11, \beta_{\text {red }}^{\mathcal{A} \widehat{\otimes} B}$ is an isomorphism. Now the assertion follows from Lemma 5.12.

Assume now that our discrete group $\Gamma$ acts properly by isometries on a locally finite space $X$ of bounded coarse geometry. As it was explained in the previous section, any proper $\Gamma$-space $Y$, such that $Y / \Gamma$ is compact, admits a $\Gamma$-equivariant map into some Rips complex $\mathfrak{M}_{k}$. As an immediate corollary of the previous proposition we get:

Corollary 5.14. Assume that for every $k \in \mathbf{R}_{+}$, there exist

- a proper $\Gamma$-algebra $\mathcal{A}_{k}$

- elements $\eta_{k} \in K K_{i}^{\Gamma}\left(\mathbf{C}, \mathcal{A}_{k}\right)$ and $d_{k} \in K K_{i}^{\Gamma}\left(\mathcal{A}_{k}, \mathbf{C}\right)$ such that $p_{\mathfrak{M}_{k}}^{*}\left(\eta_{k} \otimes_{\mathcal{A}_{k}} d_{k}\right)=1_{\mathfrak{M}_{k}}$, where $p_{\mathfrak{M}_{k}}$ is the map $\mathfrak{M}_{k} \rightarrow$ point, $p_{\mathfrak{M}_{k}}^{*}$ is the $\operatorname{map} K K^{\Gamma}(\mathbf{C}, \mathbf{C}) \rightarrow R K_{\Gamma}^{0}\left(\mathfrak{M}_{k}\right)$.

Then $\beta_{\mathrm{red}}^{B}$ is injective for every $\Gamma$-algebra $B$.

\section{The $\gamma$ element}

This section contains one of the main ingredients of the proof of Theorem 5.2. Namely, assuming that $(X, d)$ is a proper $\Gamma$-space which is locally finite, weakly geodesic, has bounded coarse geometry and satisfies a condition somewhat weaker than weak bolicity, we construct an element $\gamma_{k} \in K K_{\Gamma}(\mathbf{C}, \mathbf{C})$ such that $q^{*}\left(\gamma_{k}\right)=1_{\mathfrak{M}_{k}} \in R K_{\Gamma}^{0}\left(\mathfrak{M}_{k}\right)$ (where $q$ is the projection $\mathfrak{M}_{k} \rightarrow$ point).

We fix a metric space $(X, d)$. Here is some additional notation that we will use:

For $N \in \mathbf{R}_{+}$, let $\Delta_{N}$ denote the set of all nonempty finite subsets of $X$ of diameter $\leq N$. (Clearly, if $X$ is locally finite, $\Delta_{N}$ is a combinatorial complex, the geometric realization of which is $\mathfrak{M}_{N}$.)

For any $S \in \Delta_{N}$, set $U_{S}=\bigcap_{y \in S} \bar{B}(y, N)=\left\{z \in X, S \cup\{z\} \in \Delta_{N}\right\}$.

We begin with the following:

Lemma 6.1. Assume $(X, d)$ is weakly $\delta$-geodesic. Let $x \in X$ and $S \in \Delta_{N}$ be such that $x \notin U_{S}$. For all $z \in U_{S}, \sup \{d(z, y), y \in S\} \geq N+d\left(x, U_{S}\right)-$ $d(x, z)-2 \delta$. 
Proof. Let $\eta \in \mathbf{R}$ be such that $d(x, z)-d\left(x, U_{S}\right)<\eta$; we must prove that there exists $c \in S$ such that $d(c, y)>N-2 \delta-\eta$. As $(X, d)$ is weakly $\delta$-geodesic, there exists a point $b \in X$ such that $d(z, b) \leq \eta+2 \delta$ and $d(x, b) \leq d(x, z)-\eta$. Since $d(x, b) \leq d(x, z)-\eta<d\left(x, U_{S}\right)$, it follows that $b \notin U_{S}$; therefore there exists $c \in S, d(b, c)>N$ whence $d(z, c) \geq d(b, c)-d(z, b)>N-2 \delta-\eta$.

We now fix nonnegative real numbers $\delta, k, N$ such that $N \geq 8 k+22 \delta$ and set $\Delta=\Delta_{N}$. In the sequel of this section we assume that $(X, d)$ is weakly $\delta$-geodesic and satisfies the following condition (which is a consequence of condition (B2') of weak bolicity):

(C2) There exists a map $m: X \times X \rightarrow X$ such that if $x, y, z$ are points of $X$, then $m(x, y)$ is a $\delta$-middle point of $x, y$ and $d(m(x, y), z) \leq \max (d(x, z)$, $d(y, z))+2 \delta$. If moreover, $d(x, z) \leq N, d(y, z) \leq N$ and $d(x, y)>N$ then $d(m(x, y), z)<N-4 k-10 \delta$.

Lemma 6.2. Let $x \in X$ and $S \in \Delta$. The diameter of $\left\{z \in U_{S}, d(x, z) \leq\right.$ $\left.d\left(x, U_{S}\right)+(4 k+6 \delta)\right\}$ is $\leq N$.

Proof. If $x \in U_{S}$ the assertion is obvious since $N \geq 8 k+12 \delta$. Let $y, z \in U_{S}$ be such that $d(x, y) \leq d\left(x, U_{S}\right)+4 k+6 \delta$ and $d(x, z) \leq d\left(x, U_{S}\right)+4 k+6 \delta$; assume $d(y, z)>N$. By condition $(\mathrm{C} 2) d(x, m(y, z)) \leq d\left(x, U_{S}\right)+4 k+8 \delta$, and for every $c \in S$ we have $d(c, m(y, z))<N-4 k-10 \delta$, which is in contradiction with Lemma 6.1.

From now on, assume that $(X, d)$ is locally finite.

Lemma 6.3. Let $x \in X$ and $S, T \in \Delta$. Assume that for every $a$ in the symmetric difference of $S$ and $T$ we have $d(a, x) \leq d\left(x, U_{S}\right)+4 k+6 \delta$. Then

a) $d\left(x, U_{T}\right)=d\left(x, U_{S}\right)$.

b) For any $b$ in the symmetric difference of $U_{S}$ and $U_{T}, d(x, b) \geq d\left(x, U_{S}\right)+$ $4 k+6 \delta$.

Proof. Using induction on the cardinality of the symmetric difference of $S$ and $T$ we may assume that this symmetric difference consists of exactly one element $a$.

Assume first that $a \in T$. Then $U_{T} \subset U_{S}$; moreover, since $T=S \cup\{a\} \in \Delta$, $a \in U_{S}$. By Lemma 6.2, the set $\left\{z \in U_{S}, d(x, z) \leq d\left(x, U_{S}\right)+4 k+6 \delta\right\}$ has diameter less than $N$ and by our assumption it contains $a$. It is therefore contained in $U_{T}$. We have proved that

$$
\left\{z \in U_{S}, d(x, z) \leq d\left(x, U_{S}\right)+4 k+6 \delta\right\} \subset U_{T} \subset U_{S} ;
$$

a) and b) follow immediately. 
Assume next that $a \in S$. Then $U_{S} \subset U_{T}$.

Suppose that $d\left(x, U_{T}\right)<d\left(x, U_{S}\right)$; set $F=\left\{b \in U_{T}, d(x, b)<d\left(x, U_{S}\right)\right\}$ and let $b \in F$ be such that $d(a, b)=d(a, F)$. As $d(x, b)<d\left(x, U_{S}\right), b \notin U_{S}$, so $d(a, b)>N$. Set $b_{1}=m(a, b)$; by condition $(\mathrm{C} 2), d\left(b_{1}, x\right) \leq d(a, x)+2 \delta$, and there exists a positive real number $\varepsilon$ such that for all $y \in T, d\left(y, b_{1}\right)+\varepsilon<$ $N-4 k-10 \delta$; we may moreover assume that $2 \varepsilon+N<d(a, b)$. Let $c \in X$ be such that $d\left(b_{1}, c\right) \leq 4 k+10 \delta+\varepsilon$ and

$$
d(x, c) \leq d\left(x, b_{1}\right)-4 k-8 \delta-\varepsilon \leq d(x, a)-4 k-6 \delta-\varepsilon \leq d\left(x, U_{S}\right)-\varepsilon .
$$

Then, $c \in F$ and therefore

$$
d(a, b) \leq d(a, c) \leq d\left(a, b_{1}\right)+4 k+10 \delta+\varepsilon \leq \frac{d(a, b)}{2}+4 k+11 \delta+\varepsilon
$$

and $N<d(a, b)-2 \varepsilon \leq 8 k+22 \delta$ which contradicts our hypothesis.

Now a) is proved; we may therefore exchange the roles of $S$ and $T$; b) follows.

Lemma 6.4. Let $x \in X$ and $S \in \Delta$ satisfy $\sup \{d(x, y), y \in S\}>4 k+6 \delta$. Then $\sup \{d(x, y), y \in S\}>d\left(x, U_{S}\right)+4 k+6 \delta$.

Proof. If $S$ has one point, the assertion is true since $(X, d)$ is weakly geodesic. Assume that $\sup \{d(x, y), y \in S\} \leq d\left(x, U_{S}\right)+4 k+6 \delta$. Let $T$ be a set consisting of one point in $S$; we get a contradiction using Lemma 6.3.

Notation. For $R \in \mathbf{R}_{+}$, let $I(R)$ be the set of real numbers $r \in \mathbf{R}_{+}$such that for every quadruple $x, y, a, b$ of points of $X$ satisfying $d(x, a)+d(y, b) \geq$ $2 R-r, d(x, y) \leq r$ and $d(a, b) \leq 2 N$, one has: $d(y, a)+d(x, b) \leq d(x, a)+$ $d(y, b)+2 k$. Note that $I(R)$ is an interval in $\mathbf{R}_{+}$containing 0 .

Lemma 6.5. a) For all $R \in \mathbf{R}_{+}, k \in I(R)$.

b) If $R \leq R^{\prime} \in \mathbf{R}_{+}, I(R) \subset I\left(R^{\prime}\right)$; if $r \in \mathbf{R}_{+}$satisfies $r+2\left(R^{\prime}-R\right) \in I\left(R^{\prime}\right)$, then $r \in I(R)$.

c) If the diameter of $X$ is infinite then $\sup (I(R)) \leq \sup \{R-N, 0\}+k+$ $6 \delta \leq \sup \{R, 6 \delta\}+k$.

Proof. a) From the inequalities $d(x, b) \leq d(x, y)+d(y, b)$ and $d(y, a) \leq$ $d(x, y)+d(x, a)$ we get $d(y, a)+d(x, b) \leq d(x, a)+d(y, b)+2 d(x, y)$, from which the first assertion follows.

In $\mathrm{b})$, the first assertion is obvious. To prove the second assertion, set $r^{\prime}=r+2\left(R^{\prime}-R\right)$. If $x, y, a, b$ satisfy $d(x, a)+d(y, b) \geq 2 R-r=$ $2 R^{\prime}-r^{\prime}, d(x, y) \leq r \leq r^{\prime}, d(a, b) \leq 2 N$, then $d(y, a)+d(x, b) \leq d(x, a)+$ $d(y, b)+2 k$. Therefore, $r \in I(R)$.

c) Let $r \in I(R)$. By b), we may replace $R$ by $\sup \{R, N\}$ and $r$ by $\inf \{r, R+N\}$. We assume that $r>R-N+k+6 \delta$, and show that $r \notin I(R)$. 
Let $y, a^{\prime} \in X$ be such that $d\left(y, a^{\prime}\right) \geq R+N$. Since $X$ is weakly $\delta$-geodesic, there exists $a \in X$ such that $d(y, a) \leq R+N+2 \delta$ and $d\left(a, a^{\prime}\right) \leq d\left(y, a^{\prime}\right)-$ $(R+N)$, whence $R+N \leq d(a, y) \leq R+N+2 \delta$. Choose $x, b \in X$ such that $d(x, y) \leq r, d(x, a) \leq d(y, a)-r+2 \delta, d(a, b) \leq 2 N$ and $d(y, b) \leq d(y, a)-2 N$ $+2 \delta$. We have:

$$
\begin{aligned}
d(x, a)+d(y, b) & \geq 2 d(y, a)-d(x, y)-d(a, b) \\
& \geq 2(R+N)-r-2 N \geq 2 R-r .
\end{aligned}
$$

Now note that $d(x, b) \geq d(y, a)-d(y, b)-d(x, a), d(y, a)-d(x, a) \geq r-2 \delta$ and $d(y, a)-d(y, b) \geq 2 N-2 \delta$; whence

$$
\begin{aligned}
d(y, a)+d(x, b)-d(x, a)-d(y, b) & \geq 2(d(y, a)-d(x, a)-d(y, b)) \\
& \geq 2(r+2 N-4 \delta-d(y, a)) \\
& \geq 2(r+2 N-4 \delta-R-N-2 \delta)>2 k .
\end{aligned}
$$

Therefore $r \notin I(R)$.

For $x \in X$ and $S \in \Delta$, put $A_{S, x}=\left\{a \in U_{S}, d(x, a) \leq d\left(x, U_{S}\right)+2 \delta\right\}$ and $C_{S, x}=\{c \in S, d(x, c) \geq \max \{d(x, y), y \in S\}-2 \delta\}$.

For $x \in X, S \in \Delta$ and $r \in \mathbf{R}_{+}$, we set

$$
Y_{S, x, r}=\bigcup_{y \in \bar{B}(x, r)} A_{S, y} \quad \text { and } \quad Z_{S, x, r}=\bigcup_{y \in \bar{B}(x, r)} C_{S, y} .
$$

Remark. In order to construct the element $\gamma$, we just need to consider the sets $A_{S, x}$ and $Y_{S, x, r}$; the sets $C_{S, x}$ and $Z_{S, x, r}$ are used in the construction of the "dual Dirac element" which will be given in the next section.

Lemma 6.6. Assume that $r \in I\left(d\left(x, U_{S}\right)\right)$.

a) For all $a \in Y_{S, x, r}, d(a, x) \leq d\left(x, U_{S}\right)+2 k+2 \delta$. For all $b \in Z_{S, x, r}$, $d(b, x) \geq \max \{d(x, y), y \in S\}-2 k-2 \delta$.

b) The diameter of $Y_{S, x, r}$ is $\leq N$.

c) For all $y \in \bar{B}(x, r)$ and all $a \in Y_{S, x, r}, d(a, y) \leq d\left(y, U_{S}\right)+4 k+2 \delta$.

d) If moreover $0<\sup \{d(x, c), c \in S\}-4 k-6 \delta$, then $Y_{S, x, r} \cap Z_{S, x, r}=\emptyset$ and the distance between $Y_{S, x, r}$ and $Z_{S, x, r}$ is $\geq 2 \delta$.

Proof. a) Choose $y, z \in \bar{B}(x, r)$ such that $a \in A_{S, y}$ and $b \in C_{S, z}$. Let also $a^{\prime} \in U_{S}, b^{\prime} \in S$ be such that $d\left(x, a^{\prime}\right)=d\left(x, U_{S}\right)$ and $d\left(x, b^{\prime}\right)=\max \{d(x, c)$, $c \in S\}$.

Note that $d(x, y) \leq r, d(y, a) \geq d\left(x, U_{S}\right)-r, d\left(x, a^{\prime}\right)=d\left(x, U_{S}\right)$, the diameter of $U_{S}$ is $\leq 2 N$, and $d(y, a) \leq d\left(y, a^{\prime}\right)+2 \delta$. As $r \in I\left(d\left(x, U_{S}\right)\right)$ we have $d(x, a) \leq d\left(x, a^{\prime}\right)+d(y, a)-d\left(y, a^{\prime}\right)+2 k \leq d\left(x, a^{\prime}\right)+2 k+2 \delta$. 
Also $d(x, z) \leq r, d\left(y, b^{\prime}\right) \geq d\left(x, U_{S}\right)-r, d(x, b) \geq d\left(x, U_{S}\right)$, the diameter of $S$ is $\leq N$, and $d\left(z, b^{\prime}\right) \leq d(z, b)+2 \delta$. As $r \in I\left(d\left(x, U_{S}\right)\right)$ we have $d\left(x, b^{\prime}\right) \leq$ $d(x, b)+2 k+d\left(z, b^{\prime}\right)-d(z, b) \leq d(x, b)+2 k+2 \delta$.

b) By a), $Y_{S, x, r} \subset\left\{a \in U_{S}, d(x, a) \leq d\left(x, U_{S}\right)+2 k+2 \delta\right\}$. Hence, b) follows from Lemma 6.2.

c) Choose $a^{\prime \prime} \in U_{S}$ such that $d\left(y, a^{\prime \prime}\right)=d\left(y, U_{S}\right)$. By a), we have $d(x, a) \leq$ $d\left(x, U_{S}\right)+2 k+2 \delta \leq d\left(x, a^{\prime \prime}\right)+2 k+2 \delta$. As $r \in I\left(d\left(x, U_{S}\right)\right)$, it follows that $d(y, a)+d\left(x, a^{\prime \prime}\right) \leq d\left(y, a^{\prime \prime}\right)+d(x, a)+2 k$, whence $d(y, a) \leq d\left(y, U_{S}\right)+4 k+2 \delta$.

d) follows from a) and Lemma 6.4.

Proposition 6.7. Let $x \in X, S \in \Delta$ and $r \in I\left(d\left(x, U_{S}\right)\right)$. Then

a) $S \cup Y_{S, x, r} \in \Delta$.

Let $T \in \Delta$ be such that $S-Y_{S, x, r} \subset T \subset S \cup Y_{S, x, r}$. Then:

b) For all $y \in \bar{B}(x, r), A_{S, y}=A_{T, y}$, and $Y_{S, x, r}=Y_{T, x, r}$.

c) If moreover $r<\sup \{d(x, c), c \in S\}-4 k-6 \delta$, then for all $y \in \bar{B}(x, r)$, $C_{S, y}=C_{T, y}$, and $Z_{S, x, r}=Z_{T, x, r}$

Proof. a) is a consequence of Lemma 6.6.b).

b) It follows from 6.6.c) that for every $a$ in the symmetric difference of $S$ and $T$ and every $y \in \bar{B}(x, r)$ we have: $d(y, a) \leq d\left(y, U_{S}\right)+4 k+2 \delta$. It remains to apply 6.3.b).

In the hypothesis of $\mathrm{c}), \sup \{d(y, c), c \in S\}>4 k+6 \delta$; by Lemma 6.4, each point of $C_{S, y}$ is at a distance $>d\left(y, U_{S}\right)+4 k+4 \delta$ from $y$. Therefore, $C_{S, y} \subset T$ by 6.3.b). In the same way, $C_{T, y} \subset S$; whence $C_{S, y}=C_{T, y}$.

\section{Construction of $\gamma$}

Let $\delta, k, N$ be positive real numbers such that $N>8 k+22 \delta$ and let $(X, d)$ be a weakly $\delta$-geodesic, locally finite metric space satisfying conditions (C2) and the following:

$$
\bigcup_{R \in \mathbf{R}_{+}} I(R)=\mathbf{R}_{+}
$$

Note that, if $\delta \leq k$, condition (C1) is slightly weaker than condition (B1).

Assume now that $X$ has bounded coarse geometry, more precisely, that:

(C3) There exists a $\Gamma$-invariant measure $\mu$ on $X$ with the property that for any $R>0$ there exists $K>0$ such that for any $x \in X, \mu(\bar{B}(x, R)) \leq K$ and $\mu(B(x, \delta)) \geq 1$. 
For a nonempty subset $T$ of $X$, let $\mu_{T}$ be the measure defined in the following way: let $\widetilde{T}$ be the $\delta$-neighborhood of the set $T$ in $X$; for $x \in \widetilde{T}$ let $p_{x}$ be the probability measure equi-distributed on all points of $T$ minimizing the distance from $x$ to $T$. Set then $\mu_{T}=\int_{\widetilde{T}} p_{x} d \mu(x)$.

The map $T \mapsto \mu_{T}$ is $\Gamma$-equivariant and satisfies:

Lemma 6.8. a) For every $x \in T, \mu_{T}(B(x, 2 \delta) \cap T) \geq 1$.

b) Let $T$ and $T^{\prime}$ be subsets of $X$. The measures $\mu_{T}$ and $\mu_{T^{\prime}}$ coincide outside the $2 \delta$-neighborhood of the symmetric difference of $T$ and $T^{\prime}$.

Proof. For every $x \in T$ and any $y \in B(x, \delta)$, the measure $p_{y}$ is supported in $B(x, 2 \delta)$. Since $B(x, \delta) \subset \widetilde{T}$ and $\mu(B(x, \delta)) \geq 1$, we deduce a).

b) For every $y \in T$, the value $\mu_{T}(y)$ only depends on the measures $p_{x}$ for $x \in B(y, \delta)$. Moreover, for $x \in \widetilde{T}$, the measure $p_{x}$ is nonzero only on $T \cap B(x, \delta)$.

For $x \in X$, and $S \in \Delta$ put $r_{S, x}=\sup I\left(d\left(x, U_{S}\right)\right)-k$.

Proposition 6.9. For any $x \in X$ and $S \in \Delta$, there exist probability measures $\psi_{S, x}$ and $\theta_{S, x}$ on $X$ which depend $\Gamma$-equivariantly on the pair $(S, x)$ and have supports in $\bigcup_{r \in I\left(d\left(x, U_{S}\right)\right)} Y_{S, x, r-k}$ and $\bigcup_{r \in I\left(d\left(x, U_{S}\right)\right)} Z_{S, x, r-k}$ respectively such that:

a) For any $x, y \in X$, the functions $S \mapsto\left\|\psi_{S, x}-\psi_{S, y}\right\|_{1}$ and $S \mapsto\left\|\theta_{S, x}-\theta_{S, y}\right\|_{1}$ converge to zero outside finite sets of $\Delta$.

b) For any $x, y \in X$ such that $d(x, y) \leq k$, and any $S, T \in \Delta$ whose symmetric difference is contained in the support of $\psi_{S, x}$, we have $\psi_{S, y}=\psi_{T, y}$; if moreover $d\left(x, U_{S}\right) \geq 6 \delta$, then $\theta_{S, x}=\theta_{T, x}$, and the distance between $\operatorname{supp} \psi_{S, x}$ and $\operatorname{supp} \theta_{S, x}$ is $\geq 2 \delta$.

Proof. Let $f_{S, x, r}$ (resp. $g_{S, x, r}$ ) denote the characteristic function of $Y_{S, x, r}$ (resp. $Z_{S, x, r}$ ). Put

$$
\begin{aligned}
& \widetilde{\psi}_{S, x}=\left(f_{S, x, 0}+\int_{0}^{r_{S, x}} f_{S, x, t} d t\right) \cdot \mu_{U_{S}} \\
& \tilde{\theta}_{S, x}=\left(g_{S, x, 0}+\int_{0}^{r_{S, x}} g_{S, x, t} d t\right) \cdot \mu_{S} .
\end{aligned}
$$

Define $\psi_{S, x}$ and $\theta_{S, x}$ to be proportional to $\widetilde{\psi}_{S, x}$ and $\widetilde{\theta}_{S, x}$ and normalized to mass 1.

In order to prove property a), we will first prove that (for fixed $x, y$ ), the functions $S \mapsto\left\|\widetilde{\psi}_{S, x}-\widetilde{\psi}_{S, y}\right\|_{1}$ and $S \mapsto\left\|\widetilde{\theta}_{S, x}-\widetilde{\theta}_{S, y}\right\|_{1}$ are bounded. 
Set $d=d(x, y)$ and let $K$ denote the maximal $\mu$-volume of balls of radius $N+\delta$ in $X$. For every subset $Y$ of $X$, the $\mu_{Y}$-volume of any ball of radius $N$ in $Y$ is $\leq K$.

Note that $r \mapsto Y_{S, x, r}$ and $r \mapsto Z_{S, x, r}$ are increasing functions; therefore, $r \mapsto f_{S, x, r}$ and $r \mapsto g_{S, x, r}$ are also increasing functions. Their norm is bounded by $K$ because $S$ and $U_{S}$ sit inside a ball of radius $N$ with center at any point of $S$. Also note that $\bar{B}(y, r) \subset \bar{B}(x, r+d)$. So by definition of $Y_{S, x, r}$ and $Z_{S, x, r}$ we have $Y_{S, y, r} \subset Y_{S, x, r+d}$ and $Z_{S, y, r} \subset Z_{S, x, r+d}$. Therefore, $f_{S, y, r} \leq f_{S, x, r+d}$ and $g_{S, y, r} \leq g_{S, x, r+d}$. Using the fact that $r_{S, y} \leq r_{S, x}+2 d$ (Lemma 6.5.b), we find $\widetilde{\psi}_{S, y} \leq \widetilde{\psi}_{S, x}+f_{S, y, 0}+3 d f_{S, y, r_{S, y}}$; exchanging the roles of $x$ and $y$, we deduce $\left\|\widetilde{\psi}_{S, x}-\widetilde{\psi}_{S, y}\right\|_{1} \leq(3 d+1) K$; in the same way, $\left\|\widetilde{\theta}_{S, x}-\widetilde{\theta}_{S, y}\right\|_{1} \leq(3 d+1) K$.

Let $a \in U_{S}$ and $b \in S$ be such that $d(x, a)=d\left(x, U_{S}\right)$ and $d(x, b)=$ $\sup \{d(x, y), y \in S\}$. Note that the ball in $U_{S}$ (resp. $S$ ) with center $a$ (resp. $b$ ) and radius $2 \delta$ is contained in $A_{S, x}$ (resp. $C_{S, x}$ ). By Lemma 6.8.a), $\mu_{U_{S}}\left(A_{S, x}\right) \geq$ 1 and $\mu_{S}\left(C_{S, x}\right) \geq 1$. Therefore the norms of $\widetilde{\psi}_{S, x}$ and $\widetilde{\theta}_{S, x}$ are $\geq r_{S, x}$. Now, condition (C1) implies $\lim _{S \rightarrow \infty} r_{S, x}=\infty$; property a) follows.

We now come to property $\mathrm{b})$. Choose among $\{x, y\}$ the point which has the maximal distance to $U_{S}$ and call it $z$. As the function $R \mapsto I(R)$ is increasing and as $d(x, z) \leq k$, every point in the support of $\psi_{S, x}$ is in some $Y_{S, x, r} \subset Y_{S, z, r+k}$ where $r+k \in I\left(d\left(x, U_{S}\right)\right) \subset I\left(d\left(z, U_{S}\right)\right)$. Now $y \in \bar{B}(z, k)$; moreover, for any $y^{\prime} \in X$ such that $y=y^{\prime}$ or $d\left(y, y^{\prime}\right)<r_{S, x}$, we have

$$
d\left(y^{\prime}, z\right) \leq k+d\left(y, y^{\prime}\right) \in I\left(d\left(y, U_{S}\right)\right) \subset I\left(d\left(z, U_{S}\right)\right)
$$

By Proposition 6.7.b), $A_{y^{\prime}, S}=A_{y^{\prime}, T}$; also, the measures $\mu_{U_{S}}$ and $\mu_{U_{T}}$ coincide in $\left\{c \in U_{S}, d(z, c) \leq d\left(z, U_{S}\right)+4 k+4 \delta\right\}$ by Lemmas 6.6.a), 6.3.b) and 6.8.b); we deduce that $\psi_{S, y}=\psi_{T, y}$.

If $d\left(x, U_{S}\right) \geq 6 \delta$, then, by Lemma 6.5.c), for all $r \in I\left(d\left(x, U_{S}\right)\right)$ we have $r \leq d\left(x, U_{S}\right)+k$; therefore, $r_{S, x} \leq d\left(x, U_{S}\right)$. Note that since $x \notin U_{S}$, we have $\sup \{d(x, c), c \in S\}>N>4 k+6 \delta$; by Lemma 6.4 , we deduce that $r_{S, x}<$ $\sup \{d(x, c), c \in S\}-4 k-6 \delta$; therefore, by Proposition 6.7.c) the functions $g_{S, x, r}$ and $g_{T, x, r}$ coincide for $r<r_{S, x}$. Now, by Lemmas 6.6.d) and 6.8.b), we deduce that $\theta_{S, x}=\theta_{T, x}$. The last assertion of the proposition also follows.

Let $\lambda \in \mathfrak{M}_{k}$. Set $\psi_{S, \lambda}=\int_{X} \psi_{S, x} d \lambda(x)$. Finally (identifying measures on $X$ and elements in $\ell^{1}(X)$ - since $X$ is discrete), let $\phi_{S, \lambda} \in \ell^{2}(X)$ be the function defined by $\phi_{S, \lambda}(y)=\left(\psi_{S, \lambda}(y)\right)^{1 / 2}$.

Denote by $\left(e_{x}\right)_{x \in X}$ the canonical basis of $\ell^{2}(X)$. Let $H$ be the Hilbert subspace of $\Lambda^{*}\left(\ell^{2}(X)\right)$ spanned by $e_{x_{1}} \wedge \ldots \wedge e_{x_{n}}$ where $\left\{x_{1}, \ldots, x_{n}\right\}$ runs over $\Delta$. Let $e_{\emptyset}$ be the vacuum vector of $\Lambda^{*}\left(\ell^{2}(X)\right)$. The grading of $H$ is the opposite to the canonical one; i.e. the degree of $e_{x_{1}} \wedge \ldots \wedge e_{x_{n}}$ is $n-1$ 
(modulo 2). For $\xi \in \ell^{2}(X)$ let $c(\xi)$ be the operator on $\Lambda^{*}\left(\ell^{2}(X)\right)$ given by the Clifford multiplication by $\xi$, i.e. $c(\xi)=\operatorname{ext}(\xi)+\operatorname{int}(\xi)$, the sum of operators of (left) exterior and interior multiplication by $\xi$.

Denote by $P$ the projection of $\Lambda^{*}\left(\ell^{2}(X)\right)$ onto $H$ and let $F_{\lambda}$ be the operator on $H$ given for $\left\{x_{1}, \ldots, x_{n}\right\}=S \in \Delta$ by the formula:

$$
F_{\lambda}\left(e_{x_{1}} \wedge \ldots \wedge e_{x_{n}}\right)=P c\left(\phi_{S, \lambda}\right)\left(e_{x_{1}} \wedge \ldots \wedge e_{x_{n}}\right) .
$$

Theorem 6.10. a) For all $\lambda \in \mathfrak{M}_{k}, F_{\lambda}=F_{\lambda}^{*}$ and $1-F_{\lambda}^{2}$ is the rank-one projection onto the vector $\phi_{\text {support }(\lambda), \lambda}$.

b) For all $\lambda, \lambda^{\prime} \in \mathfrak{M}_{k}, F_{\lambda}-F_{\lambda^{\prime}} \in \mathcal{K}(H)$.

c) The map $\lambda \mapsto F_{\lambda}$ is norm-continuous on $\mathfrak{M}_{k}$.

Proof. If $S=\left\{x_{1}, \ldots x_{n}\right\}$ is a finite subset of $X$ denote by $L_{S}$ the line in $\Lambda^{*}\left(\ell^{2}(X)\right)$ spanned by $e_{x_{1}} \wedge \ldots \wedge e_{x_{n}}$.

a) Let $\lambda \in \mathfrak{M}_{k}$ and $S \in \Delta$. Let $x$ be a point in the support of $\lambda$ maximizing the distance to $U_{S}$. Since the function $R \mapsto I(R)$ is increasing, the support of $\phi_{S, \lambda}$ is contained in $\bigcup_{r \in I\left(d\left(x, U_{S}\right)\right)} Y_{S, x, r}$.

For all $S \in \Delta, c\left(\phi_{S, \lambda}\right) L_{S}$ is contained in the sum of spaces $L_{T}$ where $T$ runs over all subsets of $X$ such that the symmetric difference of $S$ and $T$ consists of exactly one point which is contained in the support of $\phi_{S, \lambda}$; in particular, $T \in \Delta \cup\{\emptyset\}$, and for every $y$ in the support of $\lambda$ we have $\psi_{S, y}=\psi_{T, y}$ if $T \neq \emptyset$ (Proposition 6.9.b). Therefore $c\left(\phi_{S, \lambda}\right)\left(L_{S}\right) \subset H \oplus \mathbf{C} e_{\emptyset}$ where $e_{\emptyset}$ is the vacuum vector of $\Lambda^{*}\left(\ell^{2}(X)\right)$, and since $c\left(\phi_{S, \lambda}\right)$ is self-adjoint, we deduce that $F_{\lambda}=F_{\lambda}^{*}$.

Consider the equivalence relation in $\Delta$ for which $S$ and $T$ are equivalent if their symmetric difference is contained in the support of $\phi_{S, \lambda}$. The vector $\phi_{S, \lambda}$ is constant on the equivalence classes. The Hilbert space $H$ breaks into an orthogonal sum of finite-dimensional subspaces spanned by the lines $L_{T}$ of equivalence classes, $F_{\lambda}$ preserves this decomposition and coincides with $c\left(\phi_{S, \lambda}\right)$ on any such class except for the class formed by all subsets $S \subset X$ such that $S \subset \operatorname{supp}\left(\phi_{S, \lambda}\right)$.

The lines $L_{S}$ for all $S$ in this class span a finite-dimensional subspace $V$ of $H$. The operator $c\left(\phi_{S, \lambda}\right)$ maps $V \oplus \mathbf{C} e_{\emptyset}$ isomorphically onto itself, so being compressed to $V$ this operator has a one-dimensional kernel. In fact, $V \oplus \mathbf{C} e_{\emptyset}$ is isomorphic to a finite-dimensional exterior algebra and $c\left(\phi_{S, \lambda}\right)$ is a Clifford multiplication operator on it. The operator $1-F_{\lambda}^{2}$ is 0 on the orthogonal complement of $V$ in $H$, and on $V$ it is a one-dimensional projection onto the image of $\mathbf{C} e_{\emptyset}$ under the Clifford multiplication by $c\left(\phi_{S, \lambda}\right)$, i.e. it is the onedimensional projection onto the vector $\phi_{S, \lambda}$. 
It remains to note that all nonempty subsets of the support of $\phi_{S, \lambda}$ belong to our distinguished class for any $S \subset \operatorname{supp}(\lambda)$. This means that the function $\phi_{S, \lambda}$ for this class is equal to $\phi_{\operatorname{support}(\lambda), \lambda}$, which establishes the expression for $1-F_{\lambda}^{2}$.

b) and c) Let $\lambda, \lambda^{\prime} \in \mathfrak{M}_{k}$; for $S \in \Delta$, put $\phi_{S}=\inf \left\{\phi_{S, \lambda}, \phi_{S, \lambda^{\prime}}\right\}$, and let $F$ be given for $\xi \in L_{S}$ by the formula $F(\xi)=P c\left(\phi_{S}\right)(\xi)$. Now, $F_{\lambda}-F$ and $F_{\lambda^{\prime}}-F$ are block diagonal. As $\left\|\phi_{S, \lambda}-\phi_{S}\right\|_{2}^{2} \leq\left\|\phi_{S, \lambda}-\phi_{S, \lambda^{\prime}}\right\|_{2}^{2} \leq\left\|\psi_{S, \lambda}-\psi_{S, \lambda^{\prime}}\right\|_{1}$, it follows from Proposition 6.9.a) that $F_{\lambda}-F$ is compact; in the same way, $F_{\lambda^{\prime}}-F$ is compact. Moreover, $\left\|F_{\lambda}-F\right\|^{2}=\sup \left\{\left\|\phi_{S, \lambda}-\phi_{S}\right\|_{2}^{2}, S \in \Delta\right\} \leq$ $\sup \left\{\left\|\psi_{S, \lambda}-\psi_{S, \lambda^{\prime}}\right\|_{1}, \quad S \in \Delta\right\} \leq\left\|\lambda-\lambda^{\prime}\right\|_{1}$; estimating (in the same way) $\left\|F_{\lambda^{\prime}}-F\right\|^{2}$, we obtain c).

The pair $\left(H, F_{x}\right)$ defines an element $\gamma=\gamma_{k}$ of $R(\Gamma)$.

Corollary 6.11. Let $q$ be the map $\mathfrak{M}_{k} \rightarrow$ point. Then $q^{*}(\gamma)=1$ in $R K_{\Gamma}^{0}\left(\mathfrak{M}_{k}\right)$.

Proof. By Theorem 6.10.a) and c), the family $\lambda \mapsto F_{\lambda}$ defines the unit element of $R K_{\Gamma}^{0}\left(\mathfrak{M}_{k}\right)$. By Theorem 6.10.b), it is equal to $q^{*}\left(\gamma_{k}\right)$.

\section{The dual Dirac construction}

In this section and the next one we finish the proof of Theorem 5.2 (and therefore of Theorem 1.1).

Recall that having a proper isometric action of a discrete group $\Gamma$ on a weakly bolic, weakly geodesic metric space of bounded coarse geometry, we can choose a discrete, locally finite subspace $X$ of this space having the same properties and still equipped with a proper isometric action of $\Gamma$ (Prop. 3.5).

The dual Dirac construction for euclidean spaces ([HKT], [HK])

Let us denote the real $L^{2}(X ; \mu)$ by $\mathcal{H}$. Recall first the construction of a $C^{*}$-algebra associated to a separable real Hilbert space ([HKT], $\left.[\mathrm{HK}]\right)$. We will consider $\mathcal{H}$ as an affine Hilbert space. For any finite-dimensional real affine subspace $V \subset \mathcal{H}$, denote by $V_{0}$ the underlying vector space. Let $C_{\tau}(V)$ be (as in $[\mathrm{K} 2], 4.1)$ the $\mathbf{Z}_{2}$-graded algebra of Clifford functions $C_{0}\left(V\right.$, Cliff $\left(V_{0}\right)$ ), where $\operatorname{Cliff}\left(V_{0}\right)$ is the complex Clifford algebra associated with the Euclidean quadratic form on $V_{0}$. (This algebra has a natural structure of a $C^{*}$-algebra.) If we fix an origin $v_{0}$ in $V$ (i.e. if we identify $V$ with $V_{0}$ ), then we can define the Bott operator $B_{v_{0}}$ for $C_{\tau}(V)$ as multiplication by the function $C: V \rightarrow \operatorname{Cliff}\left(V_{0}\right)$ whose value at $v \in V$ is $v-v_{0} \in \operatorname{Cliff}\left(V_{0}\right)$. It is a degreeone, essentially self-adjoint, unbounded multiplier of $C_{\tau}(V)$, with domain the compactly supported functions in $C_{\tau}(V)$. 
Denote by $\mathcal{S}=C_{0}(\mathbf{R})$ the $\mathbf{Z}_{2}$-graded algebra of continuous complex-valued functions on $\mathbf{R}$ which vanish at infinity. $\mathcal{S}$ is graded according to even and odd functions. We will use the following notation: $\mathcal{A}(V)=\mathcal{S} \widehat{\otimes} C_{\tau}(V)$. Denote by $X$ the operator of multiplication by $x$ on $\mathbf{R}$, viewed as a degree one, essentially self-adjoint, unbounded multiplier of $\mathcal{S}$ with domain the compactly supported functions in $\mathcal{S}$.

Suppose now that $V$ and $V^{\prime}$ are finite-dimensional affine subspaces of $H$ with $V^{\prime} \subset V$. Let $W$ be the orthogonal complement of $V^{\prime}$ in $V$. Since $W$ naturally has an origin (call it $w_{0}$ ), we can identify $W$ with $W_{0}$. Define a homomorphism $\mathcal{S} \rightarrow \mathcal{A}(W)$ by the formula: $f \mapsto f\left(X \widehat{\otimes} 1+1 \widehat{\otimes} B_{w_{0}}\right)$. Using a canonical decomposition: $\mathcal{A}(V)=\mathcal{A}(W) \widehat{\otimes} C_{\tau}\left(V^{\prime}\right)$, define a homomorphism $\mathcal{A}\left(V^{\prime}\right) \rightarrow \mathcal{A}(V)$ by tensoring the above homomorphism $\mathcal{S} \rightarrow \mathcal{A}(W)$ with the identity map on $C_{\tau}\left(V^{\prime}\right)$. These homomorphisms $\mathcal{A}\left(V^{\prime}\right) \rightarrow \mathcal{A}(V)$ are transitive with respect to the embeddings $V^{\prime \prime} \subset V^{\prime} \subset V$. The algebra $\mathcal{A}(\mathcal{H})$ is defined as the inductive limit of the algebras $\mathcal{A}(V)$ taken over the directed set of all finite-dimensional affine subspaces of $\mathcal{H}$. We will consider this $C^{*}$-algebra as trivially graded.

Note that for any point $v \in \mathcal{H}$, there exists a canonical unbounded multiplier of $\mathcal{A}(\mathcal{H})$, the unbounded Bott element $B_{v}$. It corresponds to the unbounded multiplier $X$ of the algebra $\mathcal{A}(v)=\mathcal{S}$ (where $v$ is considered as an affine subspace of $\mathcal{H}$ ) under the inductive limit map $\mathcal{A}(v) \rightarrow \mathcal{A}(\mathcal{H})$. The unbounded multiplier $B_{v}$ is essentially self-adjoint; its resolvent belongs to $\mathcal{A}(\mathcal{H})$; moreover $B_{v}-B_{w}$ is bounded for $v, w \in \mathcal{H}$, and $g B_{v}=B_{g v}$ for all $g \in \Gamma$. Therefore $B_{v}$ defines an element $\eta_{\mathcal{H}} \in K K_{1}^{\Gamma}(\mathbf{C}, \mathcal{A}(\mathcal{H}))$. Note that in our case of $\mathcal{H}=L^{2}(X ; \mu)$, the action of $\Gamma$ on $\mathcal{H}$ is linear, so the operator $B_{0}$ (corresponding to $v=0$ ) is exactly $\Gamma$-invariant.

\section{The algebra $\mathcal{A}_{k}$}

In the previous section, for any $k \in \mathbf{R}_{+}$, we have defined $N \in \mathbf{R}_{+}$. Let us fix $k$ and $N$ and consider the map $\tau: \mathfrak{M}_{N} \rightarrow L^{2}(X ; \mu)$ defined in 4.2. Denote the image of this map (with the topology induced by the weak topology of $\left.L^{2}(X ; \mu)\right)$ by $Z_{k}$. According to Lemma $4.3, Z_{k}$ is a proper $\Gamma$-space.

We will define now a $C^{*}$-algebra $\mathcal{A}_{k}$ which is a $\Gamma-Z_{k}$-algebra. Note that whereas $\mathfrak{M}_{N}$ is obviously a simplicial complex, with simplices corresponding to subsets $S \subset X$ of diameter $\leq N$, the space $Z_{k}$ has only a structure of a polyhedral complex. For any simplex $S$ in $\Delta_{N}$, we denote by $|S| \subset \mathfrak{M}_{N}$ its geometric realization (i.e. the set of probability measures on $X$ with support in $S)$; the subspace $\tau(|S|)$ is a convex polyhedron in $L^{2}(X ; \mu)$. We will denote this polyhedron by $\widetilde{S}$ and call it quasisimplex.

Let $q_{S}$ be the map of $\mathcal{H}$ into itself which associates to each point of $\mathcal{H}$ the

nearest point of its finite-dimensional subquasisimplex $\widetilde{S}$. It is clear that this 
map is well defined and continuous in the weak topology. Using this map, one can lift any continuous function on $\widetilde{S}$ to a weakly continuous function on $\mathcal{H}$. This map defines a homomorphism of the algebra of continuous functions on the quasisimplex into the algebra of weakly continuous functions on $\mathcal{H}$. But since multiplication by a bounded weakly continuous function on $\mathcal{H}$ naturally defines a central multiplier of $\mathcal{A}(\mathcal{H})$ (of degree 0 ), we obtain a homomorphism $C(\widetilde{S}) \rightarrow \mathcal{M}(\mathcal{A}(\mathcal{H}))$.

Let $H$ be the (graded) Hilbert space entering the definition of the $\gamma$ element of the previous section. The $C^{*}$-algebra $\mathcal{K}(H) \otimes \mathcal{A}(\mathcal{H})$ is endowed with the natural (diagonal) action of the group $\Gamma$. Moreover, there exists also a $\Gamma$ equivariant homomorphism of the algebra of functions $C_{0}\left(Z_{k}\right)$ to $\mathcal{M}(\mathcal{K}(H) \otimes$ $\mathcal{A}(\mathcal{H})$ ). Namely, if we fix a natural basis in $H$ as in the previous section (each basis vector corresponding to an oriented simplex of $\mathfrak{M}_{N}$ ), then the Hilbert module $H \otimes \mathcal{A}(\mathcal{H})$ over $\mathcal{A}(\mathcal{H})$ becomes a direct sum over the simplices $S$ of $\mathfrak{M}_{N}$ of the algebras $\mathcal{A}(\mathcal{H})$. For each simplex $S$, there is a homomorphism of $C(\widetilde{S}) \rightarrow \mathcal{M}(\mathcal{A}(\mathcal{H}))$ as defined above. This homomorphism clearly does not depend on the orientation of $S$, and all these homomorphisms combine together to give the homomorphism $C_{0}\left(Z_{k}\right) \rightarrow \oplus_{S} C(\widetilde{S}) \rightarrow \mathcal{L}(H \otimes \mathcal{A}(\mathcal{H}))$.

Definition 7.1. Let $\mathcal{A}_{k}$ be the subalgebra of all elements of the $C^{*}$-algebra $\mathcal{K}(H) \otimes \mathcal{A}(\mathcal{H})$ which commute with the image of the above homomorphism $C_{0}\left(Z_{k}\right) \rightarrow \mathcal{M}(\mathcal{K}(H) \otimes \mathcal{A}(\mathcal{H}))$. There exists a natural restriction of the homomorphism $C_{0}\left(Z_{k}\right) \rightarrow \mathcal{M}(\mathcal{K}(H) \otimes \mathcal{A}(\mathcal{H}))$ to a homomorphism $C_{0}\left(Z_{k}\right) \rightarrow \mathcal{M}\left(\mathcal{A}_{k}\right)$. This latter homomorphism defines a structure of a $C_{0}\left(Z_{k}\right)$ algebra on $\mathcal{A}_{k}$.

This definition requires some explanation. First of all, the algebra $\mathcal{A}_{k}$ is very similar to the algebra of a finite-dimensional simplicial complex defined in [KS1] and called $\mathcal{A}_{X}$ there. We could not use that definition here because in general the Rips complex $\mathfrak{M}_{N}$ is not finite-dimensional. The main difference between the algebra $\mathcal{A}_{X}$ of $[\mathrm{KS} 1]$ and the algebra $\mathcal{A}_{k}$ defined here is that in [KS1] we used the function called type to map simplices of our simplicial complex into a Euclidean space, whereas here we just have an embedding of our polyhedral complex $Z_{k}$ into the Hilbert space $\mathcal{H}$.

To make this parallel between the definition of [KS1] and the definition given here completely precise, let us consider the Hilbert module $H \otimes \mathcal{A}(\mathcal{H})$ over $\mathcal{A}(\mathcal{H})$ as a direct sum over the simplices $S$ of $\mathfrak{M}_{N}$ of the algebras $\mathcal{A}(\mathcal{H})$. The algebra $\mathcal{A}_{k}$ clearly contains all elements of $\mathcal{K}(H \otimes \mathcal{A}(\mathcal{H}))$ which are diagonal with respect to this decomposition. It also contains nondiagonal elements, namely for those pairs of copies of $\mathcal{A}(\mathcal{H})$ in the direct sum $\oplus_{S} \mathcal{A}(\mathcal{H})$ which correspond to quasisimplices $\widetilde{S}, \widetilde{S}^{\prime}$ with $\widetilde{S} \cap \widetilde{S}^{\prime} \neq \emptyset$. One easily verifies that $\mathcal{M}\left(\mathcal{A}_{k}\right)$ is a subalgebra of $\mathcal{L}(H \otimes \mathcal{A}(\mathcal{H}))$ and that $\mathcal{A}_{k}=\mathcal{M}\left(\mathcal{A}_{k}\right) \cap \mathcal{K}(H \otimes \mathcal{A}(\mathcal{H}))$. 
The algebra $\mathcal{A}_{k}$ just constructed is a proper $\Gamma$-algebra (Def. 5.6). This will be the algebra $\mathcal{A}_{k}$ of Corollary 5.14. In order to apply Corollary 5.14, it is enough to construct the Dirac and the dual Dirac elements $d_{k}$ and $\eta_{k}$. The Dirac element will be constructed in the next section. Here we construct the dual Dirac element $\eta_{k} \in K_{1}^{\Gamma}\left(\mathcal{A}_{k}\right)$. First we will choose for any simplex $S$ of the Rips complex a certain vector $v_{S} \in \mathcal{H}$ which will be the center of the Bott element for this simplex.

\section{The choice of the vector $v_{S}$}

In the previous section, we have associated to any simplex $S$ of the combinatorial Rips complex $\Delta_{N}$ and any point $x \in X$, a measure $\theta_{S, x} \in \mathfrak{M}_{N}$ (Prop. 6.9). Let us put $\xi_{S, x}=\tau\left(\theta_{S, x}\right)$ where $\tau$ is the map defined in 4.2.

For all $R>0$, let $K(R)>0$ denote the maximal $\mu$-measure of a subset $T \subset X$ of diameter $\leq R$. Put $K=K(N+2 \delta)$.

Lemma 7.2. Let $\rho, \alpha$ be positive real numbers such that $\alpha>2 \rho K^{3 / 2}+K^{2}$. Put $v_{S}=\alpha \xi_{S, x}$, and let $z$ be any point of $\mathcal{H}$ contained in a ball of radius $\rho$ around $v_{S}$. Suppose that a point $a \in S$ is at a distance $\geq 2 \delta$ in $X$ from the support of the function $\theta_{S, x}$. Then $q_{S}(z)$ (the nearest-to- $z$ point of the quasisimplex $\widetilde{S})$ actually belongs to the subquasisimplex $(S-\{a\})^{\sim}$ in $\mathcal{H}$.

Proof. Put $y=q_{S}(z)$. The condition that $y$ is the nearest-to- $z$ point of the quasisimplex $\widetilde{S} \subset \mathcal{H}$ means that $\widetilde{S}$ lies in the half-space which is cut off from $z$ by a hyperplane passing through the point $y$ and orthogonal to the vector $y-z$. This can be expressed by saying that for any point $s \in \widetilde{S}$, the scalar product $\langle s-y, y-z\rangle$ is nonnegative. In particular $\left\langle\xi_{S, x}-y, y-z\right\rangle \geq 0$, since $\xi_{S, x} \in \widetilde{S}$.

Let $s_{1}, \ldots, s_{m}$ be all vertices of $S$. Then $y$ is a convex linear combination of the points $\tau\left(s_{1}\right), \ldots, \tau\left(s_{m}\right)$. The nonzero coefficients in this linear combination can correspond only to those points among $\tau\left(s_{1}\right), \ldots, \tau\left(s_{m}\right)$ that lie in the hyperplane passing through the point $y$ and orthogonal to the vector $y-z$. We claim that $\left\langle\tau(a)-\xi_{S, x}, y-z\right\rangle>0$. It follows that $\langle\tau(a)-y, y-z\rangle>0$. Therefore $\tau(a)$ cannot lie in this hyperplane, and the lemma follows.

To prove our claim, note that by Lemma $4.3,\left\|\xi_{S, x}\right\| \geq K^{-1 / 2}$ and $\|y\| \leq$ $K^{1 / 2}$. Therefore

$$
\begin{aligned}
\left\langle\xi_{S, x}, z-y\right\rangle & =\left\langle\xi_{S, x}, v_{S}+\left(z-v_{S}\right)-y\right\rangle \geq \alpha\left\|\xi_{S, x}\right\|^{2}-\left\|\xi_{S, x}\right\|\left(\rho+K^{1 / 2}\right) \\
& \geq \alpha K^{-1}-K^{1 / 2}\left(\rho+K^{1 / 2}\right)
\end{aligned}
$$

Note also that an element of $\widetilde{S}$ is a nonnegative function on $X$, hence $\langle\tau(a), y\rangle \geq 0$. 
Since the point $a \in S$ is at a distance $\geq 2 \delta$ in $X$ from the support of the function $\theta_{S, x}$, one has $\left\langle\tau(a), v_{S}\right\rangle=0$. So

$$
\begin{aligned}
\left\langle\tau(a)-\xi_{S, x}, y-z\right\rangle & =\langle\tau(a), y\rangle-\left\langle\tau(a), z-v_{S}\right\rangle+\left\langle\xi_{S, x}, z-y\right\rangle \\
& \geq-\rho K^{1 / 2}+\alpha K^{-1}-K^{1 / 2}\left(\rho+K^{1 / 2}\right)>0
\end{aligned}
$$

as claimed.

We choose $\rho=1$ and put $\alpha=2\left(K^{3 / 2}+K^{2}\right)$; hence, $v_{S}=2\left(K^{3 / 2}+K^{2}\right) \xi_{S, x}$.

\section{The operator $\Phi$}

We are ready now to construct an operator $\Phi \in \mathcal{M}\left(\mathcal{A}_{k}\right)$ which will give us the required dual Dirac element in $K_{1}^{\Gamma}\left(\mathcal{A}_{k}\right)$. Let us denote the Hilbert module $H \otimes \mathcal{A}(\mathcal{H})$ over $\mathcal{A}(\mathcal{H})$ by $\mathfrak{H}$. We will consider $\mathfrak{H}$ as a direct sum over the oriented simplices $\{S\}$ of $\mathfrak{M}_{N}$ of the algebras $\mathcal{A}(\mathcal{H})$. The operator $\Phi$ will be constructed in $\mathcal{L}(\mathfrak{H})=\mathcal{M}(\mathcal{K}(H) \otimes \mathcal{A}(\mathcal{H}))$ and then we will prove that it actually belongs to $\mathcal{M}\left(\mathcal{A}_{k}\right)$.

Let $B_{v_{S}}$ be the unbounded Bott element for $\mathcal{A}(\mathcal{H})$ centered at the point $v_{S}$. Denote by $\operatorname{dim}[S]$ the dimension of the simplex $S$ in $\mathfrak{M}_{N}$. Define a function $\nu$ on the real line by $\nu(t)=t \cdot(\max (1,|t|))^{-1}$. Also let $\omega$ be a positive continuous function on $\mathbf{R}_{+}$satisfying the conditions: $\omega(t)=0$ if $t \leq 6 \delta$ and $\omega(t)=1$ if $t \geq 8 \delta$.

The operator $\Phi$ consists of a part $\Phi_{\text {diag }}$ diagonal with respect to the above direct sum decomposition of $\mathfrak{H}$ and an off-diagonal part $\Phi_{\text {off }}$. The operator $\Phi$ will depend on the point $x \in X$. When we need to show the dependence of $\Phi$ on $x$ we will denote it by $\Phi_{x}$.

Recall from the proof of Theorem 6.10 that if $S=\left\{x_{1}, \ldots x_{n}\right\}$ is a finite subset of $X$, we denote by $L_{S}$ the line in $\Lambda^{*}\left(\ell^{2}(X)\right)$ spanned by $e_{x_{1}} \wedge \ldots \wedge e_{x_{n}}$. The diagonal part $\Phi_{\text {diag }}$ of our operator is defined, for $S=\left\{x_{1}, \ldots, x_{n}\right\} \in \Delta_{N}$ and $\xi \in L_{S}$, by $\Phi_{\operatorname{diag}}(\xi \otimes \zeta)=(-1)^{\operatorname{dim}[S]-1} \xi \otimes \nu\left(B_{v_{S}}\right)(\zeta)$.

The off-diagonal part $\Phi_{\text {off }}$ takes into account the operator $F=F_{x}$ of the $\gamma$-element constructed in the previous section (here $x \in X$ is considered as the Dirac measure $\lambda$ at the point $x)$. Let $\widetilde{\omega}$ be the multiplication by $\omega\left(d\left(x, U_{S}\right)\right)$ on $L_{S} \otimes \mathcal{A}(\mathcal{H})$. We set $\Phi_{\text {off }}=(F \otimes 1)\left(1-\Phi_{\text {diag }}^{2}\right)^{1 / 2} \widetilde{\omega}$, and finally, $\Phi=\Phi_{\text {diag }}+\Phi_{\text {off }}$.

TheOREM 7.3. The element $\Phi$ is a multiplier of the algebra $\mathcal{A}_{k}$. It satisfies the following conditions: $\Phi^{*}=\Phi, 1-\Phi^{2} \in \mathcal{A}_{k}$. The element $\Phi$ depends equivariantly on the point $x \in X$, namely: $g\left(\Phi_{x}\right)=\Phi_{g x}$, for all $g \in \Gamma$, and for any $y \in X, \quad \Phi_{y}-\Phi_{x} \in \mathcal{A}_{k}$. Thus $\eta_{k}=\left(\mathcal{A}_{k}, \Phi\right)$ is an element of $K_{1}^{\Gamma}\left(\mathcal{A}_{k}\right)$. Moreover, the image of $\eta_{k}$ in the group $K_{1}^{\Gamma}(\mathcal{K}(H) \otimes \mathcal{A}(\mathcal{H}))$ under the natural embedding $\mathcal{A}_{k} \subset \mathcal{K}(H) \otimes \mathcal{A}(\mathcal{H})$ is equal to the product of the $\gamma$-element $\gamma_{k}$ defined by the operator $F$ and the Bott element $\eta_{\mathcal{H}} \in K K_{1}^{\Gamma}(\mathbf{C}, \mathcal{A}(\mathcal{H}))$. 
Proof. Obviously the operators $\widetilde{\omega}$ and $\Phi_{\text {diag }}$ commute. According to Lemmas 6.3.a) and 6.6.a), if $S, T \in \Delta_{N}$ are such that $F\left(L_{S}\right)$ and $L_{T}$ are not orthogonal, then $d\left(x, U_{S}\right)=d\left(x, U_{T}\right)$. Therefore $\widetilde{\omega}$ and $F \otimes 1$ commute. If moreover $d\left(x, U_{S}\right) \geq 6 \delta$, then according to Proposition 6.9.b) the vectors $\xi_{S, x}$ and $\xi_{T, x}$ coincide, so that $v_{S}=v_{T}$. It follows that $\Phi_{\text {diag }}$ and $(F \otimes 1) \widetilde{\omega}$ anticommute. Therefore $\Phi^{*}=\Phi$ and $1-\Phi^{2}=\left(1-\widetilde{\omega}^{2}\left(F^{2} \otimes 1\right)\right)\left(1-\Phi_{\text {diag }}^{2}\right) \in \mathcal{K}(H) \otimes \mathcal{A}(\mathcal{H})$ (recall that the space $X$ is locally finite, and the condition $d\left(x, U_{S}\right) \geq 8 \delta$ is not satisfied only for a finite number of simplices $S$ ).

The assertion concerning the equivariance of $\Phi$ is clear.

By Proposition 6.9 and Lemma 4.3, we have: $\Phi_{\text {diag, } y}-\Phi_{\text {diag, } x} \in \mathcal{K}(H)$ $\otimes \mathcal{A}(\mathcal{H})$; from Theorem 6.10 , it follows that

$$
\left(\left(F_{x}-F_{y}\right) \otimes 1\right)\left(1-\Phi_{\text {diag }, x}^{2}\right)^{1 / 2} \in \mathcal{K}(H) \otimes \mathcal{A}(\mathcal{H}) .
$$

Therefore $\Phi_{y}-\Phi_{x} \in \mathcal{K}(H) \otimes \mathcal{A}(\mathcal{H})$.

Now we have to prove that the operator $\Phi$ is a multiplier of $\mathcal{A}_{k}$. Indeed, the operator $\Phi_{\text {diag }}$ is obviously a multiplier of $\mathcal{A}_{k}$, so we need to prove only that $\Phi_{\text {off }}$ is also a multiplier of $\mathcal{A}_{k}$. Suppose that $F\left(L_{S}\right)$ and $L_{T}$ are not orthogonal. Then we have to show that the operators of multiplication by functions from $C_{0}\left(Z_{k}\right)$ are the same on the two copies of $\mathcal{H}$ corresponding to $S$ and $T$ in the regions where $1-\Phi_{\text {diag }}^{2} \neq 0$. These regions are in fact balls of radius 1 around points $v_{S}$ and $v_{T}$ respectively.

First of all, in the case when $d\left(x, U_{S}\right) \leq 6 \delta$, the operator $\Phi_{\text {off }}$ is zero by definition. Therefore, we may assume that $d\left(x, U_{S}\right) \geq 6 \delta$. According to Lemma 7.2 , applied in the case when $a$ is any point in the symmetric difference of $S$ and $T$, the projection maps for the ball of radius 1 around $v_{S}$ (or $v_{T}$ ) to $\widetilde{S}$ and to $\widetilde{T}$ are identical, and the image of these maps belongs to the intersection of $\widetilde{S}$ and $\widetilde{T}$. (Note that the condition that the point $a$ is at a distance $\geq 2 \delta$ in $X$ from the support of the function $\theta_{S, x}$ is satisfied in view of the last assertion of Proposition 6.9.) Therefore, the operators of multiplication by functions lifted

from $\widetilde{S}$ and $\widetilde{T}$, which are defined using these projections, are the same in the two balls that we consider.

The last assertion of the theorem follows from the existence of an obvious homotopy moving all centers $\left\{v_{S}\right\}$ of the Bott elements used in the construction of the operator $\Phi$ to the zero point of $\mathcal{H}$. The function $\omega$ in the process of this homotopy is replaced by 1 .

\section{End of proof of the main result}

In Section 5 we reduced the proof of Theorem 5.2 to the verification of the sufficient conditions given in Corollary 5.14. In this section we prove that these sufficient conditions are fulfilled for groups satisfying the assumptions 
of Theorems 1.1, 5.2. More precisely, we will construct a Dirac element $d_{k} \in$ $K K^{\Gamma}\left(\mathcal{A}_{k}, \mathbf{C}\right)$ such that (in the notation of Corollary 5.14), $p_{\mathfrak{M}_{k}}^{*}\left(\eta_{k} \otimes_{\mathcal{A}_{k}} d_{k}\right)$ $=1_{\mathfrak{M}_{k}}$.

Consider the extension of $C^{*}$-algebras

$$
0 \rightarrow \mathcal{K}(\mathcal{E}) \rightarrow \mathcal{F} \rightarrow \mathcal{A}(\mathcal{H}) \rightarrow 0
$$

constructed in $[\mathrm{HK}]$, where $\mathcal{E}$ is a Hilbert $D$-module. Here $D=C_{0}\left((0,1)^{2}\right)$ since we view all $C^{*}$-algebras as trivially graded. Let us tensor this extension with $\mathcal{K}(H)$ :

$$
0 \rightarrow \mathcal{K}(H) \otimes \mathcal{K}(\mathcal{E}) \rightarrow \mathcal{K}(H) \otimes \mathcal{F} \rightarrow \mathcal{K}(H) \otimes \mathcal{A}(\mathcal{H}) \rightarrow 0 .
$$

Since the algebra $\mathcal{A}_{k}$ is proper and nuclear, we can apply Proposition 5.7 to get a six term exact sequence associated with the exact sequence (2).

Definition 8.1. The Dirac element $d_{k} \in K K_{1}^{\Gamma}\left(\mathcal{A}_{k}, \mathcal{K}(H) \otimes \mathcal{K}(\mathcal{E})\right)=$ $K K_{1}^{\Gamma}\left(\mathcal{A}_{k}, \mathbf{C}\right)$ is defined to be $\partial\left(u_{k}\right)$, where $u_{k} \in K K^{\Gamma}\left(\mathcal{A}_{k}, \mathcal{K}(H) \otimes \mathcal{A}(\mathcal{H})\right)$ is associated with the embedding $\mathcal{A}_{k} \rightarrow \mathcal{K}(H) \otimes \mathcal{A}(\mathcal{H})$, and $\partial$ is the connecting map of the exact sequence (2).

Proposition 8.2. $\quad p_{\mathfrak{M}_{k}}^{*}\left(\eta_{k} \otimes_{\mathcal{A}_{k}} d_{k}\right)=1_{\mathfrak{M}_{k}}($ up to sign $)$.

Proof. The action of $\Gamma$ on $\mathfrak{M}_{k}$ is proper. Applying Remark 5.10, we find

$$
p_{\mathfrak{M}_{k}}^{*}\left(\eta_{k} \otimes_{\mathcal{A}_{k}} d_{k}\right)=p_{\mathfrak{M}_{k}}^{*}\left(\eta_{k}\right) \otimes_{\mathcal{A}_{k}} p_{\mathfrak{M}_{k}}^{*} \partial\left(u_{k}\right)=\partial\left(p_{\mathfrak{M}_{k}}^{*}\left(\eta_{k} \otimes_{\mathcal{A}_{k}} u_{k}\right)\right)
$$

where we have denoted by the same letter $\partial$ the connecting maps

$$
R K K^{\Gamma}\left(\mathfrak{M}_{k} ; \mathcal{A}_{k}, \mathcal{K}(H) \otimes \mathcal{A}(\mathcal{H})\right) \rightarrow R K K_{1}^{\Gamma}\left(\mathfrak{M}_{k} ; \mathcal{A}_{k}, \mathcal{K}(H) \otimes \mathcal{K}(\mathcal{E})\right)
$$

and

$$
R K K_{1}^{\Gamma}\left(\mathfrak{M}_{k} ; \mathbf{C}, \mathcal{K}(H) \otimes \mathcal{A}(\mathcal{H})\right) \rightarrow R K K^{\Gamma}\left(\mathfrak{M}_{k} ; \mathbf{C}, \mathcal{K}(H) \otimes \mathcal{K}(\mathcal{E})\right)
$$

associated with the exact sequence (2).

The last assertion of Theorem 7.3 implies that $\eta_{k} \otimes_{\mathcal{A}_{k}} u_{k}=\gamma_{k} \otimes_{\mathbf{C}} \eta_{\mathcal{H}}$ where $\eta_{\mathcal{H}} \in K K_{1}^{\Gamma}(\mathbf{C}, \mathcal{A}(\mathcal{H}))$ is the Bott element for $\mathcal{A}(\mathcal{H})$. By Corollary 6.11, we then find $p_{\mathfrak{M}_{k}}^{*}\left(\eta_{k} \otimes_{\mathcal{A}_{k}} u_{k}\right)=p_{\mathfrak{M}_{k}}^{*}\left(\eta_{\mathcal{H}}\right)$.

Therefore $p_{\mathfrak{M}_{k}}^{*}\left(\eta_{k} \otimes_{\mathcal{A}_{k}} d_{k}\right)=\partial\left(p_{\mathfrak{M}_{k}}^{*}\left(\eta_{\mathcal{H}}\right)\right)$ where $\partial$ is the connecting map for the exact sequence (1). Let us denote $C_{0}(0,1)$ by $S$ and consider the extension of $\Gamma$-algebras

$$
0 \rightarrow \mathcal{K}(\mathcal{E}) \rightarrow \mathcal{F}_{S} \rightarrow S \rightarrow 0
$$

which is the pull-back of the extension (1) along the natural embedding $S \rightarrow$ $\mathcal{A}(\mathcal{H})$. (This embedding $S \rightarrow \mathcal{A}(\mathcal{H})$ is defined by the embedding of the point 0 into $\mathcal{H}$ - see $\S 7$.) If $a$ is the class in $K K_{1}^{\Gamma}(\mathbf{C}, S)$ of the Bott element of $S$, then, 
according to Remark 5.10, it will be enough to prove that $\partial\left(p_{\mathfrak{M}_{k}}^{*}(a)\right)=1_{\mathfrak{M}_{k}}$ where $\partial$ now is the connecting map for the exact sequence (3).

In the proof of Theorem 6.10 in $[\mathrm{HK}]$, the extension (3) was presented as a sum of two extensions: one of them was

$$
0 \rightarrow S \otimes C_{0}(0,1) \rightarrow S \otimes C_{0}[0,1) \rightarrow S \rightarrow 0
$$

and the second was a restriction (to the point 1 of the half-interval $(0,1])$ of a certain extension

$$
0 \rightarrow C_{0}(0,1] \otimes \mathcal{K}\left(\mathcal{E}^{\prime}\right) \rightarrow \mathcal{F}_{S}^{\prime} \rightarrow S \rightarrow 0
$$

where $\mathcal{E}^{\prime}$ is another Hilbert $D$-module. Note that the connecting map $\partial$ for a sum of two extensions is the sum of connecting maps. For the extension (4) it is easy to prove directly that (up to sign) $\partial(a)=1$. On the other hand, for the extension (5), as well as for its restriction to the point 1 , the connecting map is 0 because the algebra $C_{0}(0,1] \otimes \mathcal{K}\left(\mathcal{E}^{\prime}\right)$ is contractible.

Remark 8.3. It can be proved that in fact $\eta_{k} \otimes_{\mathcal{A}_{k}} d_{k}=\gamma_{k}$. Let us indicate the main steps of this proof.

The Dirac element $d_{k} \in K K_{1}^{\Gamma}\left(\mathcal{A}_{k}, \mathbf{C}\right)$ gives rise to an (equivariant) asymptotic morphism of $\mathcal{A}_{k}$ to compact operators in some Hilbert space. Related to this asymptotic morphism, there exists (see [HK, Def. 7.7]) an associated homomorphism $\left(d_{k}\right)_{*}: K_{1}^{\Gamma}\left(\mathcal{A}_{k}\right) \rightarrow K_{1}^{\Gamma}(\mathbf{C})$ such that $\left(d_{k}\right)_{*}\left(\eta_{k}\right)=\eta_{k} \otimes_{\mathcal{A}_{k}} d_{k}$ (see Theorem 7.8 of $[\mathrm{HK}])$.

The convenience of viewing the latter product as $\left(d_{k}\right)_{*}\left(\eta_{k}\right)$ is that $\left(d_{k}\right)_{*}\left(\eta_{k}\right)$ is functorial in $d_{k}$. In fact, there exists another asymptotic morphism, which we will denote $d \otimes 1$, of $\mathcal{A}(\mathcal{H}) \otimes \mathcal{K}(H)$ to the algebra of compact operators such that the asymptotic morphism corresponding to $d_{k}$ is its restriction to $\mathcal{A}_{k}$. (Because the algebra $\mathcal{A}(\mathcal{H}) \otimes \mathcal{K}(H)$ is not proper, we cannot define a $K K$-element corresponding to this asymptotic morphism.) This allows one to replace $\left(d_{k}\right)_{*}\left(\eta_{k}\right)$ with $(d \otimes 1)_{*}\left(\eta_{\mathcal{H}} \otimes_{\mathbf{C}} \gamma_{k}\right)$ (by functoriality and Theorem 7.3). (Note that $(d \otimes 1)_{*}$ is defined only on elements of the group $K_{1}^{\Gamma}(\mathcal{A}(\mathcal{H}) \otimes \mathcal{K}(H))$ which have some special form; see [HK, Def. 7.7]. The element $\eta_{\mathcal{H}} \otimes_{\mathbf{C}} \gamma_{k}$ satisfies this condition.)

Now $d \otimes 1$ is really a product of the Dirac asymptotic morphism $d$ for $\mathcal{A}(\mathcal{H})$ (see [HK, Def. 6.7]) and the identity map on $\mathcal{K}(H)$. So

$$
(d \otimes 1)_{*}\left(\eta_{\mathcal{H}} \otimes_{\mathbf{C}} \gamma_{k}\right)=d_{*}\left(\eta_{\mathcal{H}}\right) \otimes_{\mathbf{C}} \gamma_{k} .
$$

(The proof of this equality is similar to the proof of Theorem 7.8 in [HK].) It remains to note that $d_{*}\left(\eta_{\mathcal{H}}\right)=1 \in K K^{\Gamma}(\mathbf{C}, \mathbf{C})$ by Theorem 6.10 of $[\mathrm{HK}]$, so that $(d \otimes 1)_{*}\left(\eta_{\mathcal{H}} \otimes_{\mathbf{C}} \gamma_{k}\right)=\gamma_{k}$. 


\section{Appendix}

In this appendix we prove that $K K^{\Gamma}(A, B) \simeq E^{\Gamma}(A, B)$ if $\mathrm{A}$ is a proper nuclear algebra.

Let us first briefly recall the definition of the functor $E^{\Gamma}(A, B)$ and the homomorphism $K K^{\Gamma}(A, B) \longrightarrow E^{\Gamma}(A, B)$ :

- An asymptotic morphism from $A$ to $B$ is given by a $\Gamma-C[0,1]$-algebra $D$ (where $\Gamma$ acts trivially on $[0,1]$ ) together with an extension of $\Gamma-C[0,1]$ algebras $0 \rightarrow B(0,1] \rightarrow D \rightarrow A \rightarrow 0$, where $C[0,1]$ acts on $A$ through

evaluation at 0 . Two such extensions $0 \rightarrow B(0,1] \rightarrow D_{1} \rightarrow A \rightarrow 0$ and $0 \rightarrow B(0,1] \rightarrow D_{2} \rightarrow A \rightarrow 0$, are said to give the same asymptotic morphism, if there is an isomorphism $f: D_{1} \rightarrow D_{2}$ with a commuting diagram

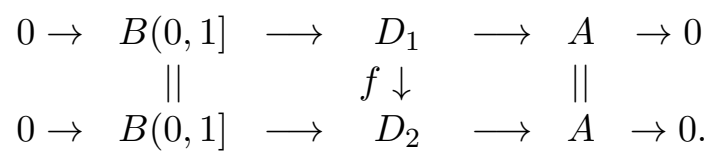

- If $x: \quad 0 \rightarrow B(0,1] \rightarrow D \rightarrow A \rightarrow 0$ is an asymptotic morphism from $A$ to $B$ and $f: B \rightarrow B_{1}$ is an equivariant $*$-homomorphism, there is an asymptotic morphism $f(x): 0 \rightarrow B_{1}(0,1] \rightarrow D_{1} \rightarrow A \rightarrow 0$ from $A$ to $B_{1}$ uniquely defined up to isomorphism by the existence of a $\Gamma-C[0,1]$ - equivariant homomorphism $g: D \rightarrow D_{1}$ such that the diagram

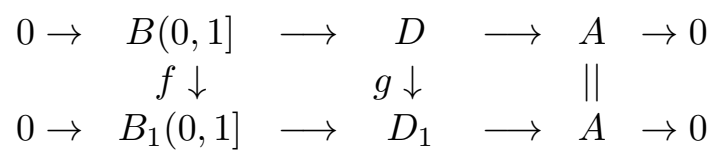

commutes. A homotopy is an asymptotic morphism from $A$ to $B[0,1]$. Two asymptotic morphisms $0 \rightarrow B(0,1] \rightarrow D_{0} \rightarrow A \rightarrow 0$ and $0 \rightarrow B(0,1] \rightarrow D_{1} \rightarrow$ $A \rightarrow 0$ from $A$ to $B$ are said to be homotopic if there exists an asymptotic morphism from $A$ to $B[0,1]$ whose evaluation at 0 is $0 \rightarrow B(0,1] \rightarrow D_{0} \rightarrow$ $A \rightarrow 0$ and whose evaluation at 1 is $0 \rightarrow B(0,1] \rightarrow D_{1} \rightarrow A \rightarrow 0$.

- Denote by $[[A, B]]$ the set of homotopy classes of asymptotic morphisms from $A$ to $B$. Denote by $[[A, B]]_{\mathrm{cp}}$ the set of homotopy classes of asymptotic morphisms from $A$ to $B$ which admit an equivariant completely positive lifting $A \rightarrow D$ of norm 1 .

- Denote by $\mathcal{K}$ the algebra of compact operators on $L^{2}(\Gamma \times \mathbf{N})$ where $\Gamma$ acts on $\Gamma$ by left translation and trivially on $\mathbf{N}$. Let also $S$ denote the $C^{*}$-algebra $C_{0}(\mathbf{R})$ (trivially graded, with a trivial action of $\Gamma$ ). We write $S A$ instead of $S \otimes A$. By definition, $E^{\Gamma}(A, B)=[[S A \otimes \mathcal{K}, S B \otimes \mathcal{K}]]$. 
- Also, according to $[\mathrm{T} 2], K K^{\Gamma}(A, B)=[[S A \otimes \mathcal{K}, S B \otimes \mathcal{K}]]_{\mathrm{cp}}$.

- The morphism $\Phi: K K^{\Gamma}(A, B) \rightarrow E^{\Gamma}(A, B)$ is defined as the forgetful map. This morphism is obviously natural in $B$ (and also in $A$, but we do not use, nor define the naturality in $A$ here).

- Tensoring with $S \mathcal{K}$ leads to well defined maps $[[A, B]] \rightarrow E^{\Gamma}(A, B)$ and $[[A, B]]_{\mathrm{cp}} \rightarrow K K^{\Gamma}(A, B)$. Tensoring again with $S \mathcal{K}$ leads to isomorphisms $E^{\Gamma}(A, B) \rightarrow E^{\Gamma}(S A \otimes \mathcal{K}, S B \otimes \mathcal{K})$ and $K K^{\Gamma}(A, B) \rightarrow K K^{\Gamma}(S A \otimes \mathcal{K}, S B \otimes \mathcal{K})$. These constructions commute with the forgetful map $\Phi$.

- The asymptotic morphism $0 \rightarrow A(0,1] \rightarrow A[0,1] \rightarrow A \rightarrow 0$ defines the unit elements $1_{A}^{K}$ of $K K^{\Gamma}(A, A)$ and $1_{A}^{E}$ of $E^{\Gamma}(A, A)$. Therefore $\Phi\left(1_{A}^{K}\right)=1_{A}^{E}$.

If $E$ is a $C_{0}(X)$-algebra and $Y \subset X$ is a closed subset, we denote by $E_{Y}$ the $C_{0}(Y)$-algebra $E / C_{0}(X-Y) E$.

Lemma A.1. Let $x: 0 \rightarrow B(0,1] \rightarrow D \rightarrow A \rightarrow 0$ be an asymptotic moprphism. Denote by $p_{0}: D \rightarrow A$ and $p_{1}: D \rightarrow B$ the evaluation maps of the $C[0,1]$-algebra $D$. There exists an asymptotic morphism $y: 0 \rightarrow D(0,1] \rightarrow \bar{D} \rightarrow A \rightarrow 0$ such that $p_{0}(y)$ is the asymptotic morphism $0 \rightarrow A(0,1] \rightarrow A[0,1] \rightarrow A \rightarrow 0$ and $p_{1}(y)=x$. If moreover $p_{0}$ admits an equivariant completely positive lifting of norm 1 , so does the evaluation map $\bar{D} \rightarrow A$.

Proof. Consider the $C^{*}$-algebra $D[0,1]=D \otimes C[0,1]$ as a $\Gamma-C([0,1] \times$ $[0,1])$-algebra: the first copy of $C[0,1]$ in $C([0,1] \times[0,1])=C[0,1] \otimes C[0,1]$ acting on $D$, the second on $C[0,1]$. Let $U=\{(s, t) \in[0,1] \times[0,1], s \leq t\}$ and $\bar{D}=D[0,1]_{U}$. Let $C[0,1]$ act on $\bar{D}$, by $f \mapsto 1 \otimes f$. In this way, $\bar{D}$ is a $C[0,1]$ algebra. We have $\bar{D}_{\{0\}}=A$. Moreover, let

$$
\varphi:[0,1] \times(0,1] \rightarrow\{(s, t) \in[0,1] \times(0,1], s \leq t\}
$$

be a homeomorphism of the form $(s, t) \mapsto(g(s, t), t)$ which is the identity in a neighborhood of $\{0\} \times(0,1]^{2}$. Extend $\varphi$ to a continuous map $[0,1] \times[0,1] \rightarrow$ $[0,1] \times[0,1]$ by the formula $\varphi(s, 0)=(0,0)$. Using $\varphi$, we get an identification of the kernel of the evaluation at $0: \bar{D} \rightarrow A$ with $D(0,1]$; we therefore get an asymptotic morphism $y: \quad 0 \rightarrow D(0,1] \rightarrow \bar{D} \rightarrow A \rightarrow 0$.

If $p_{0}$ admits an equivariant completely positive lifting $h$, then $\pi \circ i \circ h$ is the lifting for the morphism $\bar{D} \rightarrow A$, where $i: D \rightarrow D \otimes C[0,1]$ is the map $i: d \mapsto a \otimes 1$ and $\pi: D \otimes C[0,1] \rightarrow \bar{D}$ is the quotient map.

The algebra $\bar{D}$ is a $C(U)$ algebra. The asymptotic morphism $p_{0}(y)$ is given by the exact sequence $0 \rightarrow A(0,1] \rightarrow \bar{D}_{\varphi(\{0\} \times[0,1])} \rightarrow A \rightarrow 0$; it is the asymptotic morphism $0 \rightarrow A(0,1] \rightarrow A[0,1] \rightarrow A \rightarrow 0$. The asymptotic morphism

\footnotetext{
${ }^{2}$ e.g. $g(s, t)=\min \left(s, t(s+t)(1+t)^{-1}\right)$.
} 
$p_{1}(y)$ is given by the exact sequence $0 \rightarrow B(0,1] \rightarrow \bar{D}_{\varphi(\{1\} \times[0,1])} \rightarrow A \rightarrow 0$. Now, $\varphi(1, s)=(s, s)$; the quotient map $D[0,1] \rightarrow \bar{D}_{\varphi(\{1\} \times[0,1])}$ composed with $i: D \rightarrow D[0,1]$ is therefore a $C[0,1]$-linear isomorphism $D \rightarrow \bar{D}_{\varphi(\{1\} \times[0,1])}$, whence $p_{1}(y)=x$.

Lemma A.2. Let $x: 0 \rightarrow B(0,1] \rightarrow D \rightarrow A \rightarrow 0$ be an asymptotic morphism. Denote by $p_{0}: D \rightarrow A$ and $p_{1}: D \rightarrow B$ the evaluation maps of the $C[0,1]$-algebra $D$.

a) The morphism $p_{0}: D \rightarrow A$ induces an isomorphism of $E^{\Gamma}(A, D)$ onto $E^{\Gamma}(A, A)$. The class of $x$ in $E^{\Gamma}(A, B)$ is $p_{1 *}\left(\left(p_{0 *}\right)^{-1}\left(1_{A}^{E}\right)\right)$.

b) If $p_{0}$ admits a completely positive equivariant lifting of norm 1 , the morphism $p_{0}: D \rightarrow A$ induces an isomorphism of $K^{\Gamma}(A, D)$ onto $K K^{\Gamma}(A, A)$. The class of $x$ in $K K^{\Gamma}(A, B)$ is $p_{1 *}\left(\left(p_{0 *}\right)^{-1}\left(1_{A}^{K}\right)\right)$.

Proof. The functor $B \longrightarrow E^{\Gamma}(A, B)$ is 'half exact' and the functor $B \longrightarrow$ $K K^{\Gamma}(A, B)$ is 'half exact' with respect to exact sequences which admit a completely positive equivariant lifting of norm 1 ([BS]). Moreover, the ideal $B(0,1]$ is contractible in an equivariant way; the first assertions in a) and b) follow. The second ones are immediate consequences of Lemma A.1.

Proposition A.3. Let $A$ be a $\Gamma$-algebra. If the functor $B \longrightarrow K K^{\Gamma}(A, B)$ is 'half exact', then the homomorphism $\Phi: K K^{\Gamma}(A, B) \longrightarrow E^{\Gamma}(A, B)$ is an isomorphism for any $\Gamma$-algebra $B$.

Proof. We just have to show that the homomorphism

$$
\Phi: K K^{\Gamma}(S A \otimes \mathcal{K}, S B \otimes \mathcal{K}) \longrightarrow E^{\Gamma}(S A \otimes \mathcal{K}, S B \otimes \mathcal{K})
$$

is an isomorphism. Since

$$
K K^{\Gamma}(S A \otimes \mathcal{K}, B)=K K^{\Gamma}(A, S B \otimes \mathcal{K}),
$$

the functor $B \longrightarrow K K^{\Gamma}(S A \otimes \mathcal{K}, B)$ is half exact. We may therefore assume that there exist $\Gamma$-algebras $A_{1}$ and $B_{1}$ such that $A=S A_{1} \otimes \mathcal{K}$ and $B=S B_{1} \otimes \mathcal{K}$; therefore $E^{\Gamma}(A, B)=[[A, B]]$ and $K K^{\Gamma}(A, B)=[[A, B]]_{\mathrm{cp}}$.

Let $x: 0 \rightarrow B(0,1] \rightarrow D \rightarrow A \rightarrow 0$ be an asymptotic morphism from $A$ to $B$. Since the ideal $B(0,1]$ is contractible in an equivariant way, the morphism $p_{0}: D \rightarrow A$ induces an isomorphism of $K K^{\Gamma}(A, D)$ onto $K K^{\Gamma}(A, A)$. Let also $p_{1}: D \rightarrow B$ be the evaluation map. To the asymptotic morphism $x$, we associate the element $\left(p_{1}\right)_{*} \circ\left(p_{0}\right)_{*}^{-1}\left(1_{A}^{K}\right) \in K K^{\Gamma}(A, B)$.

The $K K$-elements associated with homotopic asymptotic morphisms coincide, since $K K^{\Gamma}$ is homotopy invariant. In this way, we obtain a homomorphism $\Psi: E^{\Gamma}(A, B) \rightarrow K K^{\Gamma}(A, B)$. 
Let $x: 0 \rightarrow B(0,1] \rightarrow D \rightarrow A \rightarrow 0$ be an asymptotic morphism from $A$ to $B$ such that $p_{0}$ admits a completely positive equivariant lifting of norm 1 . The class of $x$ in $K K^{\Gamma}(A, B)$ is $\left(p_{1}\right)_{*} \circ\left(p_{0}\right)_{*}^{-1}\left(1_{A}^{K}\right)$ (Lemma A.2.b). Therefore $\Psi \circ \Phi$ is the identity ( since $\left.\Phi\left(1_{A}^{E}\right)=1_{A}^{K}\right)$.

Let $x: 0 \rightarrow B(0,1] \rightarrow D \rightarrow A \rightarrow 0$ be an asymptotic morphism from $A$ to $B$. As $\Phi\left(1_{A}^{E}\right)=1_{A}^{K}$ and $\Phi$ is natural in $B$, it follows that $\Phi(\Psi(x))=$ $\Phi\left(\left(p_{1}\right)_{*} \circ\left(p_{0}\right)_{*}^{-1}\left(1_{A}^{K}\right)\right)=\left(p_{1}\right)_{*} \circ\left(p_{0}\right)_{*}^{-1}\left(\Phi\left(1_{A}^{K}\right)\right)$ which is the class of $x$ in $E^{\Gamma}(A, B)$ (Lemma A.2.a). It follows that $\Phi \circ \Psi$ is the identity.

Using Proposition 5.7 we obtain now:

Corollary A.4. Let $A$ be a nuclear proper $\Gamma$-algebra. Then $\Phi: K K^{\Gamma}(A, B)$ $\rightarrow E^{\Gamma}(A, B)$ is an isomorphism.

Remarks A.5. a) Note that the converse of Proposition A.3 is obviously true: since the functor $B \longrightarrow E^{\Gamma}(A, B)$ is 'half exact', if the homomorphism $K K^{\Gamma}(A, B) \longrightarrow E^{\Gamma}(A, B)$ is an isomorphism for every $\Gamma$-algebra $B$, then the functor $B \longrightarrow K K^{\Gamma}(A, B)$ is 'half exact'.

b) It follows that the Baum-Connes conjecture in $E$-theory of [GHT] is equivalent to the one in $K K$-theory.

Institut de Mathématiques de Luminy, Marseille, France

E-mail address: kasparov@iml.univ-mrs.fr FRANCE

Institut de Mathématiques de Jussieu, Université Denis Diderot (Paris ViI), Paris,

E-mail address: skandal@math.jussieu.fr

\section{REFERENCES}

[BCH] P. Baum, A. Connes, and N. Higson, Classifying space for proper actions and $K$ theory of group $C^{*}$-algebras, Contemp. Math. 167 (1994), 240-291.

[BK] M. Bucher and A. KARLsson, On the definition of bolic spaces, Expositiones Math. 20 (2002), 269-277.

[BS] S. BaAj et G. Skandalis, $C^{*}$-algèbres de Hopf et théorie de Kasparov équivariante, K-Theory 2 (1989), 683-721.

[BT] F. Bruhat and J. Tits, Groupes réductifs sur un corps local. I. Donnée radicielles valuées, Publ. Math. IHES 41 (1972), 5-251.

[CM] A. Connes and H. Moscovici, Cyclic cohomology, the Novikov conjecture and hyperbolic groups, Topology 29 (1990), 345-388.

[CGM] A. Connes, M. Gromov, and H. Moscovici, Group cohomology with Lipschitz control and higher signatures, Geom. Funct. Anal. 3 (1993), 1-78.

[G1] M. Gromov, Spaces and questions. (Tel Aviv, 1999), Geom. Funct. Anal. 2000 (Special Volume, Part I), 118-161.

[G2] Random walk in random groups, preprint I.H.E.S., 2002.

[GHT] E. Guentner, N. Higson, and J. Trout, Equivariant E-theory for $C^{*}$-algebras, Mem. Amer. Math. Soc. 148 (2000), A. M. S., Providence, RI.

[H] S. Helgason, Differential Geometry and Symmetric Spaces, Academic Press, New York, 1962. 
[HK] N. Higson and G. Kasparov, $E$-theory and $K K$-theory for groups which act properly and isometrically on Hilbert space, Invent. Math. 144 (2001), 23-74.

[HKT] N. Higson, G. Kasparov, and J. Trout, A Bott periodicity theorem for infinitedimensional Euclidean space, Adv. Math. 135 (1998), 1-40.

[HLS] N. Higson, V. Lafforgue, and G. Skandalis, Counterexamples to the Baum-Connes conjecture, Geom. Funct. Anal. 12 (2002), 330-354.

[HR] N. Higson and J. Roe, On the coarse Baum-Connes conjecture, London Math. Soc. Lecture Note Ser. 227 (1995), 227-254.

[JV] P. Julg and A. VAlette, Fredholm modules associated to Bruhat-Tits buildings, Proc. Centre Math. Anal., Australian Nat. Univ. (Canberra, 1987) 16 (1988), 143155.

[K1] G. Kasparov, Hilbert $C^{*}$-modules: theorems of Stinespring and Voiculescu, $J . O p$ erator Theory 4 (1980), 133-150.

[K2] Equivariant $K K$-theory and the Novikov conjecture, Invent. Math. 91 (1988), 147-201.

[KS1] G. Kasparov and G. Skandalis, Groups acting on buildings, operator $K$-theory, and Novikov's conjecture, K-Theory 4 (1991), 303-337.

[KS2] Groupes "boliques" et conjecture de Novikov, C. R. Acad. Sci. Paris Sér. I Math. 319 (1994), 815-820.

[L] V. Lafforgue, $K$-théorie bivariante pour les algèbres de Banach et conjecture de Baum-Connes, Invent. Math. 149 (2002), 1-95.

[P] N. C. Phillips, Equivariant K-theory for Proper Actions, Pitman Res. Notes in Math. Ser. 178, Longman Scientific \& Technical, Harlow, and John Wiley \& Sons, New York, 1989.

[S] G. Skandalis, Exact sequences for the Kasparov groups of graded algebras, Canad. J. Math. 37 (1985), 193-216.

[T1] K. Thomsen, Asymptotic equivariant E-theory. I, preprint.

[T2] Asymptotic homomorphisms and equivariant KK-theory, J. Funct. Anal. 163 (1999), 324-343.

[Tu] J. L. Tu, La conjecture de Baum-Connes pour les feuilletages moyennables, $K$ Theory 17 (1999), 215-264.

(Received February 23, 2001) 